\title{
Bounded solutions and their asymptotics for a doubly nonlinear Cahn-Hilliard system
}

\author{
ElEnA BOnETTI \\ e-mail: elena.bonetti@unimi.it \\ Pierluigi Colli \\ e-mail: pierluigi.colli@unipv.it \\ LUCA SCARPA \\ e-mail: luca.scarpa@univie.ac.at \\ Giuseppe TOMAssetTI \\ e-mail: giuseppe.tomassetti@uniroma3.it \\ (1) Dipartimento di Matematica "F.Enriques", Università degli Studi di Milano \\ Via Saldini 50, 20133 Milano, Italy \\ (2) Dipartimento di Matematica "F. Casorati", Università di Pavia \\ Via Ferrata 5, 27100 Pavia, Italy \\ (3) Faculty of Mathematics, University of Vienna \\ Oskar-Morgenstern-Platz 1, 1090 Vienna, Austria \\ (4) Dipartimento di Ingegneria - Sezione Ingegneria Civile \\ Università degli Studi "Roma Tre", Via Vito Volterra 62, Roma, Italy
}

\begin{abstract}
In this paper we deal with a doubly nonlinear Cahn-Hilliard system, where both an internal constraint on the time derivative of the concentration and a potential for the concentration are introduced. The definition of the chemical potential includes two regularizations: a viscosity and a diffusive term. First of all, we prove existence and uniqueness of a bounded solution to the system using a nonstandard maximumprinciple argument for time-discretizations of doubly nonlinear equations. Possibly including singular potentials, this novel result brings improvements over previous approaches to this problem. Secondly, under suitable assumptions on the data, we show the convergence of solutions to the respective limit problems once either of the two regularization parameters vanishes.
\end{abstract}

AMS Subject Classification: 35B25, 35D35, 35G31, 35K52, 74N20, 74N25.

Key words and phrases: Cahn-Hilliard equation, nonlinear viscosity, non-smooth regularization, nonlinearities, initial-boundary value problem, bounded solutions, asymptotics. 


\section{Introduction}

The main focus of this paper is the asymptotic behaviour, when either of the positive parameters $\varepsilon$ or $\delta$ converges to zero, of the following system:

$$
\begin{aligned}
\partial_{t} u-\Delta \mu=0 & \text { in } \Omega \times(0, T), \\
\mu \in \varepsilon \partial_{t} u+\beta\left(\partial_{t} u\right)-\delta \Delta u+\psi^{\prime}(u)+g & \text { in } \Omega \times(0, T), \\
\partial_{\mathbf{n}} u=0, \quad \mu=0 & \text { in } \partial \Omega \times(0, T), \\
u(0)=u_{0} & \text { in } \Omega,
\end{aligned}
$$

where $\Omega \subset \mathbb{R}^{3}$ is a smooth bounded domain and $T>0$ is a fixed final time. Here $\beta$ is a maximal monotone graph, $\psi^{\prime}$ is the derivative of a possibly non-convex potential, and $g$ is a forcing term. We shall address the unknowns $u$ and $\mu$ as, respectively, the concentration and the chemical potential.

System (1.1)-(1.2) is a modification of the celebrated Cahn-Hilliard (C-H) system, a phenomenological model that has its origin in the work of J.W. Cahn [8] concerning the effects of interfacial energy on the stability of spinodal states in solid binary solutions. Cahn's work built upon previous collaboration with J.W. Hilliard [9], where the functional

$$
\mathcal{F}(u)=\int_{\Omega}\left(\psi(u)+\frac{\delta}{2}|\nabla u|^{2}\right)
$$

was proposed as a model for the (Helmholtz) free energy of a non-uniform system whose composition is described by the scalar field $u$. In this functional, the bulk energy $\psi(u)$ represents the specific energy of a uniform solution, typically a non-convex function. The quadratic gradient energy $\frac{\delta}{2}|\nabla u|^{2}$ takes into account microscopic mechanisms that penalize spatial variation of composition, and that are responsible for the presence of interfacial energy between phases at the macroscopic scale. Cahn showed that certain states, which would be unstable if only the bulk energy was accounted for, are in fact stable under local perturbations, when the gradient energy is included in the picture.

Besides being a fundamental contribution to Materials Science, the C-H system has had considerable success in many other branches of Science and Engineering where segregation of a diffusant leads to pattern formation, such as population dynamics [20], image processing [6], dynamics for mixtures of fluids [16], tumor modelling [1,12,13], to name a few.

In the derivation of the Cahn-Hilliard system, the variation of the free energy (1.5), namely,

$$
\mu_{\mathrm{C}-\mathrm{H}}:=\frac{\delta \mathcal{F}}{\delta u}(u)=\psi^{\prime}(u)-\delta \Delta u,
$$

is the chemical potential that drives the space-time evolution of the concentration $u$ through the diffusion equation (1.1). Here we have written it after rescaling time, so that the mobility (which we assume to be constant) is numerically equal to the unity (equivalently, one may look at the Cahn-Hilliard system as the gradient flow, with respect to the norm of the dual of a Sobolev space [15]). The connection between (1.1)-(1.2) and the $\mathrm{C}-\mathrm{H}$ system is more transparent if we rewrite (1.2) as a pair of an equation and an inclusion:

$$
\mu=\mu_{\mathrm{C}-\mathrm{H}}+\varepsilon \partial_{t} u+\xi, \quad \xi \in \beta\left(\partial_{t} u\right)
$$


The additional terms on the right-hand side do not affect the energy, but rather the dissipation. This is evident from the energetic estimate

$$
\frac{d}{d t} \mathcal{F}(u(t))+\int_{\Omega}|\nabla \mu|^{2}+\left(\varepsilon \partial_{t} u+\beta\left(\partial_{t} u\right)\right) \partial_{t} u \leq-\int_{\Omega} g \partial_{t} u
$$

which is obtained by testing the first equation by $\mu$, the second equation by $-\partial_{t} u$, and by adding the resulting equations.

Since the original work of Cahn, innumerable generalizations of the C-H system have been proposed in the literature. They are so many that it would be difficult to provide a comprehensive account in the present context. We prefer to refer to the review [24]. In this respect it is worth mentioning that a systematic procedure to derive and generalize the C-H system has been proposed by M.E. Gurtin [17], by extending the thermodynamical framework of continuum mechanics, as also reported in [21]. Let us also mention an alternative approach due to Podio-Guidugli [27] leading to another viscous $\mathrm{C}-\mathrm{H}$ system of nonstandard type [10,11].

In this sea of literature, the problem that we consider belongs to the class of doublynonlinear Cahn-Hilliard systems, characterized by nonlinearity both on the instantaneous value $u$ of the concentration and on its time derivative $\partial_{t} u$. The particular form (1.1) -(1.2) has been the object of mathematical investigation in [22] with Neumann homogeneous conditions for the chemical potential, and in a previous paper of ours [5], where a discussion of its thermodynamical consistency can also be found. The system (1.1)-(1.2) has also been studied in [29] under dynamic boundary conditions. A similar system was investigated in [23], where the nonlinearity $\beta\left(\partial_{t} u\right)$ is replaced by $\partial_{t} \alpha(u)$. Among other mathematical work on the $\mathrm{C}-\mathrm{H}$ system related to the present paper, we mention the contributions by Novick-Cohen and al. 25, 26] on the viscous $\mathrm{C}-\mathrm{H}$ equation, which is obtained in the case $\beta=0$ removing the nonlinear viscosity contribution.

In all of the above-mentioned results, existence of solutions for the system (1.1)-(1.2) is proved under some polynomial growth assumptions either on the nonlinearity $\beta$ acting on the viscosity or on the nonlinearity $\psi$. While this is certainly satisfactory in providing some first existence results, on the other hand it would be desirable to obtain well-posedness for the system even for possibly singular choices of the nonlinearities. Indeed, this is not only interesting from the mathematical perspective, but especially in the direction of applications: it is well-known in fact that the most physically-relevant choice for the double-well potential $\psi$ is the so-called logarithmic one, defined as

$$
\begin{aligned}
& \psi_{\log }(r):=\frac{c}{2}[(1+r) \ln (1+r)+(1-r) \ln (1-r)]-\frac{c_{0}}{2} r^{2}, \quad r \in(-1,1), \\
& \text { with } 0<c<c_{0} .
\end{aligned}
$$

The first main question that we answer in this paper concerns then the well-posedness of system (1.1)-(1.4) in the case of arbitrarily singular nonlinearities $\beta$ and $\psi$. Our first main result (see Theorem 2.2) is a proof of the existence and uniqueness of bounded solutions for the system (1.1)-(1.4) under no growth assumptions on $\beta$ and $\psi$, possibly including logarithmic behaviours as above. In this direction, we are inspired by some arguments performed in [4], covering the analysis of the system (1.1)-(1.4) in the singular case $\delta=0$. The main idea here was based on the fact that if the initial condition is within 
a finite interval (contained in the effective domain of the potential $\psi$ ) and if the bulk free energy has sufficiently fast growth, then the concentration is essentially bounded in the parabolic domain $Q_{T}=\Omega \times(0, T)$. This allowed to deduce, through the Gronwall lemma, a contraction estimate to prove existence and uniqueness of solutions. However, in our case the presence of the term $-\delta \Delta u$ in the inclusion for the chemical potential prevents us from relying on a similar contraction argument. To overcome this problem, we prove a preliminary boundedness result: using a maximum principle for doubly nonlinear parabolic equations in combination with a suitable time-discretization of the problem, we show that the solution $u$ never touches the edges of the domain of $\psi$ and remains bounded in the parabolic domain $Q_{T}$. Thus, we are able to prove well-posedness also with very singular behaviours of $\psi$ and $\beta$, under less stringent conditions on the potential than those in [5]. This novel result actually improves the previous approaches to the problem; moreover, the argument is not standard at all and, in our opinion, gives value to our contribution.

Once well-posedness is established in this general framework, we focus on questions of more qualitative nature. More specifically, both the viscous term and the energetic term in (1.2) provide assistance in handling the possible non-smoothness of $\beta$ and the nonlinearity of $\psi^{\prime}$. It is then natural to inquire whether one of these terms, alone, is sufficient to guarantee well-posedness, and whether the singular limits obtained when either $\varepsilon \searrow 0$ or $\delta \searrow 0$ converge to the the limiting equations.

The second main result of this paper (see Theorem 2.4) is an asymptotic result, and shows convergence of the solutions of (1.1) -(1.4) in the limit $\varepsilon \searrow 0$, with $\delta>0$ being fixed. This confirms that the diffusive regularization $-\delta \Delta u$ alone allows to handle the doubly nonlinear problem, even when the nonlinearity $\beta$ acting on the viscosity is multivalued and not necessarily coercive. For example, a physically relevant choice for $\beta$ in connection with phase-change and Stefan-type problems is the multivalued graph

$$
\beta_{\text {sign }}(r):= \begin{cases}-1 & \text { if } r<0 \\ {[-1,1]} & \text { if } r=0 \\ 1 & \text { if } r>0\end{cases}
$$

Note that although $\beta_{\text {sign }}$ is nonsmooth and noncoercive, it can be chosen in the equation (1.2) as long as $\delta>0$ only (even for $\varepsilon=0$ ). From the mathematical perspective, the main tools that we use here are compactness arguments combined with monotone analysis techniques in order to pass to the limit in the two nonlinearities.

An alternative scenario to handle the monotone term would be to accompany it with the viscous regularization $\varepsilon \partial_{t} u$ alone, discarding the energetic regularization $-\delta \Delta u$ through the interface energy. The degenerate case $\delta=0$ was the object of the investigation in [4. This belongs to a wider class of degenerate parabolic systems which find their application in the modelling of hysteretic behaviour in diffusion process, such as hysteresis in porous media [2, 7, 30, 32] or in hydrogen storage devices [18]. In all these cases, the major manifestation of hysteresis is in the fact that the pressure that is needed to induce adsorption is higher than the pressure needed to induce desorption. This scenario is the object of our third Theorem [2.6, which covers the asymptotics of the system (1.1) -(1.2) as $\delta \searrow 0$, with $\varepsilon>0$ being fixed. The main tools that we rely on consist again in compactness and monotonicity techniques: furthermore, in the asymptotics $\delta \searrow 0$ we 
are able to show some refined $L^{\infty}$-estimates, allowing us to prove also the convergence rate as $\delta \searrow 0$.

Note that if in addition to $\delta=0$ we assume also $\beta=0$, then we recover the viscous forward-backward parabolic equation studied in [26]. The asymptotics $\delta \searrow 0$ in the viscous case $\varepsilon>0$ and with $\beta=0$ was studied in the work [14, where convergence of the vioscous Cahn-Hilliard to the limiting forward-backward parabolic equation was proved.

Here is the outline of the paper. In the next section we state the precise assumptions, the analytical setting, and the main theorems that we prove. In Section 3, we prove the existence result for $\delta, \varepsilon>0$ generalizing the results in [5]. Then in Sections 4 and 5 we perform the asymptotics investigation once we let vanish the approximating paramaters $\varepsilon$ and $\delta$, respectively.

\section{Assumptions and main results}

Throughout the paper, $\Omega$ is a smooth bounded domain in $\mathbb{R}^{3}$ with boundary $\Gamma$ and $T>0$ is a fixed final time; for any $t \in(0, T]$ we use the notation

$$
Q_{t}:=\Omega \times(0, t), \quad \Sigma_{t}:=\Gamma \times(0, t), \quad Q:=Q_{T}, \quad \Sigma:=\Sigma_{T} .
$$

Moreover, we introduce the spaces

$$
\begin{aligned}
& H:=L^{2}(\Omega), \quad V:=H^{1}(\Omega), \quad V_{0}:=H_{0}^{1}(\Omega), \\
& W=H^{2}(\Omega), \quad W_{0}=W \cap V_{0}, \quad W_{\mathbf{n}}:=\left\{v \in W: \partial_{\mathbf{n}} v=0 \text { a.e. on } \Gamma\right\},
\end{aligned}
$$

endowed with their usual norms, and we identify $H$ with its dual, so that $\left(V, H, V^{*}\right)$ is a Hilbert triplet. The symbol $\langle\cdot, \cdot\rangle$ denotes the duality pairing between $V^{*}$ and $V$. We will need the following lemma, which is a variation of the well-know compactness lemma (see e.g. [19, Lem. 5.1, p. 58]).

Lemma 2.1. For every $\sigma>0$, there exists $C_{\sigma}>0$ such that

$$
\|z\|_{H}^{2} \leq \sigma\|\nabla z\|_{H}^{2}+C_{\sigma}\|z\|_{V_{0}^{*}}^{2} \quad \forall z \in V .
$$

Proof. By contradiction, assume that there is $\bar{\sigma}>0$ and a sequence $\left(z_{n}\right)_{n} \subseteq V$ such that

$$
\left\|z_{n}\right\|_{H}^{2}>\bar{\sigma}\left\|\nabla z_{n}\right\|_{H}^{2}+n\left\|z_{n}\right\|_{V_{0}^{*}}^{2} \quad \forall n \in \mathbb{N} .
$$

Then, setting $v_{n}:=z_{n} /\left\|z_{n}\right\|_{H}$ (note that $z_{n} \neq 0$ for all $n$ ), it follows immediately that

$$
\left\|v_{n}\right\|_{H}=1, \quad \bar{\sigma}\left\|\nabla v_{n}\right\|_{H}^{2}+n\left\|v_{n}\right\|_{V_{0}^{*}}^{2}<1 \quad \forall n \in \mathbb{N} .
$$

Consequently, we deduce that there is $v \in H$ and $w \in H^{N}$ such that, as $n \rightarrow \infty$,

$$
v_{n} \rightarrow v \quad \text { in } H, \quad \nabla v_{n} \rightarrow w \quad \text { in } H^{N}, \quad v_{n} \rightarrow 0 \quad \text { in } V_{0}^{*} .
$$

The first two convergences imply that $v \in V, w=\nabla v$ and $v_{n} \rightarrow v$ in $V$. Since $V \stackrel{c}{\hookrightarrow} H$ is compact, we deduce that $v_{n} \rightarrow v$ in $H$. Moreover, from the third convergence and the 
fact that $H \hookrightarrow V_{0}^{*}$ continuously, we infer that $v=0$. However, by the strong convergence in $H$ we have

$$
0=\|v\|_{H}=\lim _{n \rightarrow \infty}\left\|v_{n}\right\|_{H}=1
$$

which is absurd. This concludes the proof.

We assume that

$$
\begin{gathered}
\psi \in C^{2}(a, b), \quad-\infty \leq a<b \leq+\infty \\
\psi(r) \geq 0 \quad \forall r \in(a, b), \\
\lim _{r \rightarrow a^{+}} \psi^{\prime}(r)=-\infty, \quad \lim _{r \rightarrow b^{-}} \psi^{\prime}(r)=+\infty \\
\psi^{\prime \prime}(r)>-K \quad \forall r \in(a, b),
\end{gathered}
$$

for a positive constant $K$. It is convenient to introduce

$$
\gamma:(a, b) \rightarrow \mathbb{R}, \quad \gamma(r):=\psi^{\prime}(r)+K r, \quad r \in \mathbb{R}
$$

which is maximal monotone and strictly increasing. In particular, there exists a unique $r_{0} \in(a, b)$ such that $\gamma\left(r_{0}\right)=0$. We also define the proper convex function

$$
\widehat{\gamma}(r):=\int_{r_{0}}^{r} \gamma(s) d s, \quad r \in(a, b) .
$$

Furthermore, let

$$
\widehat{\beta}: \mathbb{R} \rightarrow[0,+\infty] \quad \text { convex and l.s.c., with } \widehat{\beta}(0)=0, \quad \beta:=\partial \widehat{\beta}
$$

and note that $0 \in \beta(0)$. We shall denote the convex conjugate of $\widehat{\beta}$ by $\widehat{\beta^{-1}}$. Note that $\widehat{\beta^{-1}}: \mathbb{R} \rightarrow[0,+\infty]$ with $\widehat{\beta^{-1}}(0)=0$, and $\partial \widehat{\beta^{-1}}$ is nothing but $\beta^{-1}$, the inverse graph of $\beta$. Let us also recall the Young inequality:

$$
r s \leq \widehat{\beta}(r)+\widehat{\beta^{-1}}(s) \quad \forall r, s \in \mathbb{R}, \quad \text { where the equality holds if and only if } s \in \beta(r)
$$

For general results on convex analysis we refer to [3].

In this setting, existence of solution for problem (1.1)-(1.4) has been shown in [5] for $\varepsilon, \delta>0$ fixed, with additional growth restrictions either on $\beta$ or $\psi$. The first main theorem that we prove here is a generalized existence result for the problem (1.1)-(1.4) with $\varepsilon, \delta>0$ fixed under no growth restrictions on the operators.

Theorem 2.2. Let $\varepsilon>0, \delta>0$, and

$$
\begin{aligned}
u_{0, \varepsilon \delta} \in W_{\mathbf{n}}, \quad & \exists\left[a_{0}, b_{0}\right] \subset(a, b): a_{0} \leq u_{0, \varepsilon \delta} \leq b_{0} \quad \text { a.e. in } \Omega, \\
& g_{\varepsilon \delta} \in H^{1}(0, T ; H) \cap L^{\infty}(Q) .
\end{aligned}
$$


Then, there are two constants $a_{0}^{\prime}, b_{0}^{\prime} \in \mathbb{R}$, possibly depending on $\varepsilon$ and $\delta$, with $\left[a_{0}, b_{0}\right] \subseteq$ $\left[a_{0}^{\prime}, b_{0}^{\prime}\right] \subset(a, b)$, and a unique triplet $\left(u_{\varepsilon \delta}, \mu_{\varepsilon \delta}, \xi_{\varepsilon \delta}\right)$ such that

$$
\begin{gathered}
u_{\varepsilon \delta} \in W^{1, \infty}(0, T ; H) \cap H^{1}(0, T ; V) \cap L^{\infty}\left(0, T ; W_{\mathbf{n}}\right) \\
a_{0}^{\prime} \leq u_{\varepsilon \delta} \leq b_{0}^{\prime} \quad \text { a.e. in } Q, \\
\mu_{\varepsilon \delta} \in L^{\infty}\left(0, T ; W_{0}\right) \cap L^{2}\left(0, T ; H^{3}(\Omega)\right), \\
\xi_{\varepsilon \delta} \in L^{\infty}(0, T ; H), \quad \psi^{\prime}(u) \in L^{\infty}(Q), \\
\xi_{\varepsilon \delta} \in \beta\left(\partial_{t} u_{\varepsilon \delta}\right) \quad \text { a.e. in } Q, \\
\partial_{t} u_{\varepsilon \delta}(t)-\Delta \mu_{\varepsilon \delta}(t)=0 \quad \text { for a.e. } t \in(0, T), \\
\mu_{\varepsilon \delta}(t)=\varepsilon \partial_{t} u_{\varepsilon \delta}(t)+\xi_{\varepsilon \delta}(t)-\delta \Delta u_{\varepsilon \delta}(t)+\psi^{\prime}\left(u_{\varepsilon \delta}(t)\right)+g(t) \quad \text { for a.e. } t \in(0, T), \\
u_{\varepsilon \delta}(0)=u_{0} .
\end{gathered}
$$

A continuous dependence result follows then.

Theorem 2.3. Let $\varepsilon>0$ and $\delta>0$. For any sets of data $\left(u_{0, i}, g_{i}\right), i=1,2$, satisfying (2.9) -(2.10), let $\left(u_{i}, \mu_{i}, \xi_{i}\right)$ denote any corresponding solutions to (2.11) $-(2.18)$. Then, there exists a constant $C_{\varepsilon \delta}$, depending on the data, such that

$$
\begin{aligned}
& \left\|\mu_{1}-\mu_{2}\right\|_{L^{2}\left(0, T ; V_{0}\right)}^{2}+\left\|u_{1}-u_{2}\right\|_{H^{1}(0, T ; H) \cap L^{\infty}(0, T ; V)}^{2}+\int_{Q}\left(\xi_{1}-\xi_{2}\right)\left(\partial_{t} u_{1}-\partial_{t} u_{2}\right) \\
& \leq C_{\varepsilon \delta}\left(\left\|u_{0,1}-u_{0,2}\right\|_{V}^{2}+\left\|g_{1}-g_{2}\right\|_{L^{2}(0, T ; H)}^{2}\right) .
\end{aligned}
$$

At this point, we state our first asymptotic result, keeping $\delta>0$ fixed and letting $\varepsilon$ tend to 0.

Theorem 2.4. Let $\delta>0$ be fixed and assume that

$$
\begin{gathered}
u_{0} \in W_{\mathbf{n}}, \quad \psi^{\prime}\left(u_{0}\right) \in H, \quad g \in H^{1}(0, T ; H), \quad g(0) \in L^{\infty}(\Omega), \\
\exists C_{1}, C_{2}>0: \quad \psi(r) \geq C_{1}|r|^{2}-C_{2} \quad \forall r \in D(\psi), \\
z_{0}:=-\delta \Delta u_{0}+\psi^{\prime}\left(u_{0}\right)+g(0) \quad \text { is such that } \widehat{\beta^{-1}}\left(-z_{0}\right) \in L^{1}(\Omega), \\
(a, b)=\mathbb{R}, \quad \exists M>0: \quad\left|\psi^{\prime \prime}(r)\right| \leq M\left(1+|r|^{5}\right) \quad \forall r \in \mathbb{R} .
\end{gathered}
$$

Let also $\left(g_{\varepsilon}\right)_{\varepsilon} \subset H^{1}(0, T ; H) \cap L^{\infty}(Q)$ fulfill

$$
g_{\varepsilon}(0)=g(0), \quad g_{\varepsilon} \rightarrow g \quad \text { in } H^{1}(0, T ; H) .
$$

Then, if $\left(u_{\varepsilon}, \mu_{\varepsilon}, \xi_{\varepsilon}\right)_{\varepsilon>0}$ denotes the unique family solving (2.11)-(2.18) with respect to the data $\left(u_{0}, g_{\varepsilon}\right)$, there exists a triplet $(u, \mu, \xi)$ such that

$$
\begin{gathered}
u \in W^{1, \infty}\left(0, T ; V_{0}^{*}\right) \cap H^{1}(0, T ; V) \cap L^{\infty}\left(0, T ; W_{\mathbf{n}}\right), \\
\mu \in L^{\infty}\left(0, T ; V_{0}\right) \cap L^{2}\left(0, T ; H^{3}(\Omega)\right), \\
\xi \in L^{\infty}(0, T ; H), \quad \psi^{\prime}(u) \in L^{\infty}(0, T ; H), \\
\xi \in \beta\left(\partial_{t} u\right) \quad \text { a.e. in } Q, \\
\partial_{t} u(t)-\Delta \mu(t)=0 \quad \text { for a.e. } t \in(0, T), \\
\mu(t)=\xi(t)-\delta \Delta u(t)+\psi^{\prime}(u(t))+g(t) \quad \text { for a.e. } t \in(0, T), \\
u(0)=u_{0} .
\end{gathered}
$$


and a sequence $\left(\varepsilon_{n}\right)_{n}$ such that, as $n \rightarrow \infty, \varepsilon_{n} \searrow 0$ and

$$
\begin{gathered}
u_{\varepsilon_{n}} \stackrel{*}{\rightarrow} u \quad \text { in } W^{1, \infty}\left(0, T ; V_{0}^{*}\right) \cap L^{\infty}\left(0, T ; W_{\mathbf{n}}\right), \quad u_{\varepsilon_{n}} \rightarrow u \quad \text { in } H^{1}(0, T ; V), \\
\mu_{\varepsilon_{n}} \stackrel{*}{\rightarrow} \mu \quad \text { in } L^{\infty}\left(0, T ; V_{0}\right), \quad \mu_{\varepsilon_{n}} \rightarrow \mu \quad \text { in } L^{2}\left(0, T ; H^{3}(\Omega)\right), \\
\xi_{\varepsilon_{n}} \stackrel{*}{\rightarrow} \xi \quad \text { in } L^{\infty}(0, T ; H), \quad \psi^{\prime}\left(u_{\varepsilon_{n}}\right) \stackrel{*}{\rightarrow} \psi^{\prime}(u) \quad \text { in } L^{\infty}(0, T ; H), \\
\varepsilon_{n} \partial_{t} u_{\varepsilon_{n}} \rightarrow 0 \quad \text { in } L^{\infty}(0, T ; H) .
\end{gathered}
$$

Furthermore, if instead of (2.23) we assume that

$$
D(\beta)=\mathbb{R}, \quad \exists M>0: \quad|s| \leq M(1+|r|) \quad \forall r \in \mathbb{R}, \quad \forall s \in \beta(r)
$$

then the same conclusion is true replacing $L^{\infty}$ with $L^{2}$ in (2.25), (2.27), (2.32) and (2.34).

Remark 2.5. Let us comment on the construction of a possible family $\left(g_{\varepsilon}\right)_{\varepsilon}$ satisfying (2.24). Since $g(0) \in L^{\infty}(\Omega)$, for instance one can choose $g_{\varepsilon}:=T_{\varepsilon}(g)$, where $T_{\varepsilon}: \mathbb{R} \rightarrow \mathbb{R}$ is the usual truncation operator at level $1 / \varepsilon$, i.e., $T_{\varepsilon}(r):=\max \{\min \{r, 1 / \varepsilon\},-1 / \varepsilon\}$ for $r \in \mathbb{R}$. Indeed, it is not difficult to check that $g_{\varepsilon}(0)=g(0)$ provided that $\frac{1}{\varepsilon}>\|g(0)\|_{L^{\infty}(\Omega)}$ and $g_{\varepsilon} \rightarrow g$ in $H^{1}(0, T ; H)$.

The second asymptotic result investigates the behavior of the system as $\delta \searrow 0$. In this case, we can prove the convergence of the whole sequence and even an error estimate in terms of $\delta$ (see (2.50) $)$.

Theorem 2.6. Let $\varepsilon>0$ be fixed. Assume

$$
\begin{gathered}
u_{0} \in H, \quad \exists\left[a_{0}, b_{0}\right] \subseteq(a, b): a_{0} \leq u_{0} \leq b_{0} \quad \text { a.e. in } \Omega, \\
g \in H^{1}(0, T ; H) \cap L^{\infty}(Q) .
\end{gathered}
$$

Let $\left(u_{0 \delta}\right)_{\delta} \subset W_{\mathbf{n}}$ and $\left(g_{\delta}\right)_{\delta} \subset H^{1}(0, T ; H) \cap L^{2}(0, T ; V) \cap L^{\infty}(Q)$ be such that

$$
\begin{gathered}
a_{0} \leq u_{0 \delta} \leq b_{0} \quad \text { a.e. in } \Omega, \\
\delta^{1 / 2}\left\|\nabla u_{0 \delta}\right\|^{2}+\delta^{3 / 2}\left\|\Delta u_{0 \delta}\right\|_{H}^{2}+\left\|\psi^{\prime}\left(u_{0 \delta}\right)\right\|_{H}^{2} \leq C, \\
\left\|g_{\delta}\right\|_{H^{1}(0, T ; H) \cap L^{\infty}(Q)}+\delta^{1 / 2}\left\|g_{\delta}\right\|_{L^{2}(0, T ; V)}^{2} \leq C, \\
u_{0, \delta} \rightarrow u_{0} \quad \text { in } H, \quad g_{\delta} \rightarrow g \quad \text { in } H^{1}(0, T ; H) .
\end{gathered}
$$

Then, if $\left(u_{\delta}, \mu_{\delta}, \xi_{\delta}\right)_{\delta>0}$ denotes the unique family solving (2.11)-(2.18) with respect to the data $\left(u_{0 \delta}, g_{\delta}\right)$, there exist a triplet $(u, \mu, \xi)$ and an interval $\left[a_{0}^{\prime}, b_{0}^{\prime}\right] \subset(a, b)$ such that

$$
\begin{gathered}
u \in W^{1, \infty}(0, T ; H) \cap L^{\infty}(Q), \quad a_{0}^{\prime} \leq u \leq b_{0}^{\prime} \quad \text { a.e. in } Q, \\
\mu \in L^{\infty}\left(0, T ; W_{0}\right), \\
\xi \in L^{\infty}(0, T ; H), \quad \psi^{\prime}(u) \in L^{\infty}(Q), \\
\xi \in \beta\left(\partial_{t} u\right) \quad \text { a.e. in } Q, \\
\partial_{t} u(t)-\Delta \mu(t)=0 \quad \text { for a.e. } t \in(0, T), \\
\mu(t)=\varepsilon \partial_{t} u(t)+\xi(t)+\psi^{\prime}(u(t))-g(t) \quad \text { for a.e. } t \in(0, T), \\
u(0)=u_{0}
\end{gathered}
$$


and, as $\delta \searrow 0$,

$$
\begin{gathered}
u_{\delta} \stackrel{*}{\rightarrow} u \quad \text { in } W^{1, \infty}(0, T ; H) \cap L^{\infty}(Q), \quad u_{\delta} \rightarrow u \quad \text { in } H^{1}(0, T ; H), \\
\mu_{\delta} \stackrel{*}{\rightarrow} \mu \quad \text { in } L^{\infty}\left(0, T ; W_{0}\right), \quad \mu_{\delta} \rightarrow \mu \quad \text { in } L^{2}\left(0, T ; V_{0}\right), \\
\psi^{\prime}\left(u_{\delta}\right) \stackrel{*}{\rightarrow} \psi^{\prime}(u) \quad \text { in } L^{\infty}(Q), \quad \psi^{\prime}\left(u_{\delta}\right) \rightarrow \psi^{\prime}(u) \quad \text { in } L^{2}(0, T ; H), \\
\xi_{\delta} \stackrel{*}{\rightarrow} \xi \quad \text { in } L^{\infty}(0, T ; H), \\
\delta^{1 / 2} u \rightarrow 0 \quad \text { in } H^{1}(0, T ; V), \quad \delta u_{\delta} \rightarrow 0 \quad \text { in } L^{\infty}\left(0, T ; W_{\mathbf{n}}\right) .
\end{gathered}
$$

In particular, there exists a constant $M>0$, independent of $\delta$, such that

$$
\begin{aligned}
& \left\|\mu_{\delta}-\mu\right\|_{L^{2}\left(0, T ; V_{0}\right)}+\left\|u_{\delta}-u\right\|_{H^{1}(0, T ; H)} \\
& \quad \leq M\left(\delta^{1 / 4}+\left\|u_{0 \delta}-u_{0}\right\|_{H}+\left\|g_{\delta}-g\right\|_{L^{2}(0, T ; H)}\right) .
\end{aligned}
$$

Remark 2.7. Note that the limit problem with $\delta=0$ admits a unique solution, as it is proved in [4, Theorem 2.1]. This result, and in particular [4, estimate (2.9)], are related to the error estimate (2.50) stated here and can be compared with the continuous dependence estimate (2.19) for $\varepsilon, \delta>0$. Actually, we point out that here, in order to prove Theorem 2.3, we are using some stronger assumptions on the initial datum depending on the fact that we deal with spatial regularity for $\delta>0$.

Remark 2.8. Let us show that, under the assumptions (2.37)-(2.38), two sequences $\left(u_{0 \delta}\right)_{\delta}$ and $\left(g_{\delta}\right)_{\delta}$ with the properties above always exist. Specifically, to construct them it is possible to employ a singular perturbation technique. Indeed, we could introduce the solution $u_{0 \delta}$ of the elliptic problem

$$
\begin{cases}u_{0 \delta}-\delta^{1 / 2} \Delta u_{0 \delta}=u_{0} & \text { in } \Omega, \\ \partial_{\mathbf{n}} u_{0 \delta}=0 & \text { on } \Gamma\end{cases}
$$

and let $g_{\delta}$ be the solution of

$$
\begin{cases}g_{\delta}(t)-\delta^{1 / 2} \Delta g_{\delta}(t)=g(t) & \text { in } \Omega \\ \partial_{\mathbf{n}} g_{\delta}(t)=0 & \text { on } \Gamma\end{cases}
$$

for all $t \in[0, T]$. Then, (2.39) follows from (2.37) and the maximum principle, while (2.40) can be shown by testing the equation in (2.51) by $u_{0 \delta}$ and subsequently comparing the terms and recalling the assumption (2.2). Also, the verification of (2.41) and (2.42) is not difficult, in particular for (2.42) one can take advantage of the properties

$$
\underset{\delta \searrow 0}{\limsup }\left\|u_{0 \delta}\right\|_{H}^{2} \leq\left\|u_{0}\right\|_{H}^{2}, \quad \limsup _{\delta \searrow 0}\left\|g_{\delta}\right\|_{H^{1}(0, T ; H)}^{2} \leq\|g\|_{H^{1}(0, T ; H)}^{2} .
$$

Remark 2.9. The regularities $u_{0} \in V$ and $g \in L^{2}(0, T ; V)$ imply $u \in H^{1}(0, T ; V)$ also for $\delta=0$. Indeed, as it is discussed in in [4, Remark 5.1] we can formally take the gradient of the equation (2.48) and test it by $\partial_{t} u$ : using the Lipschitz continuity of the operator $(I+\beta)^{-1}$ (where $I$ denotes the identity) and the Gronwall lemma, it is straightforward to infer that $u \in H^{1}(0, T ; V)$ (see [4, Remark 5.1] for details). 


\section{Proof of Theorems $2.2 \longleftarrow 2.3$}

This section is devoted to the proof of the above mentioned results.

\subsection{The existence result}

We focus here on the proof of Theorem 2.2. The main idea is to approximate the problem as in [5] and to show that the approximated solutions satisfy further refined uniform estimates. As $\delta$ and $\varepsilon$ are fixed positive numbers in this section, we shall consider with no restriction that $\varepsilon=\delta=1$. Moreover, in order to simplify the presentation, we shall avoid the subscripts $\varepsilon$ and $\delta$ for $g$ and $u_{0}$.

Let now $\left(g_{\lambda}\right)_{\lambda \in(0,1)} \subseteq H^{1}(0, T ; H) \cap L^{2}(0, T ; V) \cap L^{\infty}(Q)$ such that

$$
g_{\lambda} \rightarrow g \quad \text { in } H^{1}(0, T ; H) \quad \text { as } \lambda \searrow 0, \quad\left\|g_{\lambda}\right\|_{L^{\infty}(Q)} \leq\|g\|_{L^{\infty}(Q)} \quad \forall \lambda \in(0,1) .
$$

For example, one can take (cf. (2.52) $) g_{\lambda}$ as the unique solution to the elliptic problem

$$
\begin{cases}g_{\lambda}-\lambda \Delta g_{\lambda}=g & \text { in } \Omega, \\ \partial_{\mathbf{n}} g_{\lambda}=0 & \text { on } \Gamma .\end{cases}
$$

Furthermore, denote by $T_{\lambda}: \mathbb{R} \rightarrow \mathbb{R}$ the truncation operator at level $1 / \lambda$, already defined in Remark 2.5. Then, reasoning as in [5] we know that there exist a unique pair $\left(u_{\lambda}, \mu_{\lambda}\right)$ such that

$$
\begin{gathered}
u_{\lambda} \in C^{1}([0, T] ; H) \cap H^{1}(0, T ; V) \cap C^{0}\left([0, T] ; W_{\mathbf{n}}\right) \cap L^{2}\left(0, T ; H^{3}(\Omega)\right), \\
\mu_{\lambda} \in C^{0}\left([0, T] ; W_{0}\right) \cap L^{2}\left(0, T ; H^{3}(\Omega)\right)
\end{gathered}
$$

and, for every $t \in[0, T]$,

$$
\begin{aligned}
& \partial_{t} u_{\lambda}(t)-\Delta \mu_{\lambda}(t)=0, \\
& \mu_{\lambda}(t)=\partial_{t} u_{\lambda}(t)+\beta_{\lambda}\left(\partial_{t} u_{\lambda}(t)\right)-\Delta u_{\lambda}(t)+\lambda u_{\lambda}(t) \\
& +\gamma_{\lambda}\left(u_{\lambda}(t)\right)-K T_{\lambda}(I+\lambda \gamma)^{-1}\left(u_{\lambda}(t)\right)+g_{\lambda}(t), \\
& u_{\lambda}(0)=u_{0} \text {, }
\end{aligned}
$$

where $\gamma$ is defined in (2.6) and $\gamma_{\lambda}, \beta_{\lambda}$ denote the Yosida approximations of the maximal monotone graphs $\gamma$ and $\beta$, respectively. Note that (3.3)-(3.5) is indeed an approximation of the original system (2.16) $-(2.18)$ in the following sense. The term $\lambda u_{\lambda}$ represents a (small) elliptic regularization that is going to vanish as $\lambda \searrow 0$. Moreover, since $T_{\lambda}$ and $(I+\lambda \gamma)^{-1}$ converge to the identity in $(a, b)$, the contribution $-K T_{\lambda}(I+\lambda \gamma)^{-1}\left(u_{\lambda}\right)$ represents an approximation of $-K u$, hence the terms $\gamma_{\lambda}\left(u_{\lambda}\right)-K T_{\lambda}(I+\lambda \gamma)^{-1}\left(u_{\lambda}\right)$ provide an approximation of $\psi^{\prime}(u)$.

The first estimates can be obtained with no additional effort from the arguments in [5, $\S 5.1-5.2]$ and owing to the Lipschitz-continuity of $T_{\lambda}$ and $(I+\lambda \gamma)^{-1}$ on $\mathbb{R}$. In particular, we can test (3.3) by $\mu_{\lambda}$, (3.4) by $\partial_{t} u_{\lambda}$, and sum. Secondly, we can also (formally) test (3.3) by $\partial_{t} \mu_{\lambda}$, the time derivative of (3.4) by $\partial_{t} u_{\lambda}$, and sum. Then, by also comparing the 
terms in (3.3) and using the elliptic regularity theory (as in [5, $\S 5.1-5.2]$ ), it is readily seen that

$$
\left\|u_{\lambda}\right\|_{W^{1, \infty}(0, T ; H) \cap H^{1}(0, T ; V)}+\left\|\mu_{\lambda}\right\|_{L^{\infty}\left(0, T ; W_{0}\right) \cap L^{2}\left(0, T ; H^{3}(\Omega)\right)} \leq c
$$

for a positive constant $c$, independent of $\lambda$.

We show now that $u_{\lambda}$ satisfies also an $L^{\infty}$-estimate by proving a maximum principle that arises from a time-discretization of the approximated problem. We shall need the following result, for which we refer to [28, Prop. 11.6].

Proposition 3.1. Let $\Phi: V \rightarrow[0,+\infty]$ and $\Xi: H \rightarrow \mathbb{R}$ be proper, convex, lower semicontinuous, and assume that there exist $c_{0}, c_{1}, c_{2}>0$ such that

$$
\begin{array}{lll}
\langle w, v\rangle \geq c_{0}\|v\|_{H}^{2} & \forall v \in H, & \forall w \in \partial \Xi(v), \\
\|w\|_{H} \leq c_{1}\left(1+\|v\|_{H}\right) & \forall v \in H, \quad \forall w \in \partial \Xi(v), \\
\langle w, v\rangle \geq c_{2}\|v\|_{V}^{2} & \forall v \in V, \quad \forall w \in \partial \Phi(v) .
\end{array}
$$

Set $A_{1}:=\partial \Phi$, let $A_{2}: H \rightarrow H$ be Lipschitz-continuous and define $A:=A_{1}+A_{2}$. Moreover, let $f \in L^{2}(0, T ; H)$ and $v_{0} \in V \cap D(\Phi)$. For every $N \in \mathbb{N}$ sufficiently large, we set $\tau:=T / N$ and consider the discretized problem

$$
\partial \Xi\left(\frac{v_{\tau}^{k}-v_{\tau}^{k-1}}{\tau}\right)+A\left(v_{\tau}^{k}\right) \ni f^{k}, \quad k=1, \ldots, N, \quad v_{\tau}^{0}=v_{0}
$$

with

$$
f^{k}=\frac{1}{\tau} \int_{(k-1) \tau}^{k \tau} f(s) d s, \quad k=1, \ldots, N .
$$

Then, problem (3.7) admits a solution $\left(v_{\tau}^{k}\right)_{k=0, \ldots, N}$, and the piecewise affine interpolants $v_{\tau}$ of $\left(v_{\tau}^{k}\right)_{k=0, \ldots, N}$ satisfy

$$
\left\|v_{\tau}\right\|_{H^{1}(0, T ; H) \cap L^{\infty}(0, T ; V)} \leq c
$$

for a positive constant $c$ independent of $\tau$. Furthermore, there are a subsequence $\left(\tau_{i}\right)_{i \in \mathbb{N}}$, with $\tau_{i} \rightarrow 0$ and an element $v \in H^{1}(0, T ; H) \cap L^{\infty}(0, T ; V)$, such that $v_{\tau_{i}} \stackrel{*}{\rightarrow} v$ in $H^{1}(0, T ; H) \cap L^{\infty}(0, T ; V)$ and $v$ is a solution to the problem

$$
\partial \Xi\left(\partial_{t} v\right)+A(v) \ni f, \quad v(0)=v_{0} .
$$

Now, note that equation (3.4) can be written as

$$
\left(I+\beta_{\lambda}\right)\left(\partial_{t} u_{\lambda}\right)+\left(-\Delta+\gamma_{\lambda}+\lambda I-K T_{\lambda}(I+\lambda \gamma)^{-1}\right)\left(u_{\lambda}\right)=\mu_{\lambda}-g_{\lambda} .
$$

Hence, for any $\lambda \in(0,1)$ fixed, we can apply Proposition 3.1 with the choices

$$
\begin{aligned}
& \Xi(v):=\frac{1}{2}\|v\|_{H}^{2}+\int_{\Omega} \widehat{\beta}_{\lambda}(v), \quad v \in H, \quad \Phi(v):=\frac{1}{2} \int_{\Omega}\left(|\nabla v|^{2}+\lambda\left|v^{2}\right|\right), \quad v \in V, \\
& A_{1}:=-\Delta+\lambda I, \quad A_{2}:=\gamma_{\lambda}-K T_{\lambda}(I+\lambda \gamma)^{-1}, \quad f:=\mu_{\lambda}-g_{\lambda}, \quad v_{0}:=u_{0, \varepsilon \delta} .
\end{aligned}
$$

Let then $\left(u_{\lambda, \tau}^{k}\right)_{k=0, \ldots, N}$ be a Rothe-sequence for the approximated problem with parameter $\lambda$. Then, since the solution $u_{\lambda}$ to (3.3)-(3.5) is uniquely determined, setting $u_{\lambda, \tau}$ as the piecewise affine interpolant of $\left(u_{\lambda, \tau}^{k}\right)_{k=0, \ldots, N}$, it turns out that

$$
u_{\lambda, \tau} \stackrel{*}{\rightarrow} u_{\lambda} \quad \text { in } H^{1}(0, T ; H) \cap L^{\infty}(0, T ; V)
$$


for the whole sequence $\left(u_{\lambda, \tau}\right)_{\tau}$.

Thanks to the estimate on $\left(\mu_{\lambda}\right)_{\lambda}$ and the boundedness of $\left(g_{\lambda}\right)_{\lambda}$, there exists a positive constant $M$, independent of $\lambda$, such that

$$
\left\|\mu_{\lambda}-g_{\lambda}\right\|_{L^{\infty}(Q)} \leq M
$$

By the growth assumption on $\psi^{\prime}$, there are $\bar{a}, \bar{b} \in \mathbb{R}$ with $r_{0} \in(\bar{a}, \bar{b}),\left[a_{0}, b_{0}\right] \subseteq[\bar{a}, \bar{b}] \subset(a, b)$, and

$$
\begin{gathered}
\psi^{\prime}(r) \geq M+1 \quad \text { for all } r \in[\bar{b}, b), \\
\psi^{\prime}(r) \leq-M-1 \quad \text { for all } r \in(a, \bar{a}] .
\end{gathered}
$$

Setting now $a_{0}^{\prime}:=\bar{a}-\frac{\bar{a}-a}{2}$ and $b_{0}^{\prime}:=\bar{b}+\frac{b-\bar{b}}{2}$, we have $\left[a_{0}, b_{0}\right] \subseteq[\bar{a}, \bar{b}] \subset\left[a_{0}^{\prime}, b_{0}^{\prime}\right] \subset(a, b)$. By the properties of the resolvent $(I+\lambda \gamma)^{-1}: \mathbb{R} \rightarrow \mathbb{R}$, it is well known that

$$
\lim _{\lambda \searrow 0}(I+\lambda \gamma)^{-1}\left(a_{0}^{\prime}\right)=a_{0}^{\prime}, \quad \lim _{\lambda \searrow 0}(I+\lambda \gamma)^{-1}\left(b_{0}^{\prime}\right)=b_{0}^{\prime}
$$

Note also that, since $\gamma\left(r_{0}\right)=0$, it holds $(I+\lambda \gamma)^{-1}\left(r_{0}\right)=r_{0}$, hence, recalling that $(I+\lambda \gamma)^{-1}$ is 1-Lipschitz-continuous,

$$
\begin{aligned}
\left|(I+\lambda \gamma)^{-1}\left(a_{0}^{\prime}\right)-r_{0}\right| & =\left|(I+\lambda \gamma)^{-1}\left(a_{0}^{\prime}\right)-(I+\lambda \gamma)^{-1}\left(r_{0}\right)\right| \leq\left|a_{0}^{\prime}-r_{0}\right| \\
\left|(I+\lambda \gamma)^{-1}\left(b_{0}^{\prime}\right)-r_{0}\right| & =\left|(I+\lambda \gamma)^{-1}\left(b_{0}^{\prime}\right)-(I+\lambda \gamma)^{-1}\left(r_{0}\right)\right| \leq\left|b_{0}^{\prime}-r_{0}\right| .
\end{aligned}
$$

Since $r_{0} \in\left(a_{0}^{\prime}, b_{0}^{\prime}\right)$, we deduce from the last inequalities that $(I+\lambda \gamma)^{-1}\left(a_{0}^{\prime}\right) \geq a_{0}^{\prime}$ and $(I+\lambda \gamma)^{-1}\left(b_{0}^{\prime}\right) \leq b_{0}^{\prime}$. Then, by making use of (3.13), we conclude that there exists $\lambda_{0} \in(0,1)$ such that, for every $\lambda \in\left(0, \lambda_{0}\right)$,

$$
a_{0}^{\prime} \leq(I+\lambda \gamma)^{-1}\left(a_{0}^{\prime}\right) \leq \bar{a}, \quad \bar{b} \leq(I+\lambda \gamma)^{-1}\left(b_{0}^{\prime}\right) \leq b_{0}^{\prime} .
$$

Moreover, since the resolvent $(I+\lambda \gamma)^{-1}$ is non-decreasing, for every $\lambda \in\left(0, \lambda_{0}\right)$ we have

$$
(I+\lambda \gamma)^{-1}(r) \leq \bar{a} \quad \forall r \in\left(a, a_{0}^{\prime}\right], \quad(I+\lambda \gamma)^{-1}(r) \geq \bar{b} \quad \forall r \in\left[b_{0}^{\prime}, b\right) .
$$

We claim now that if the initial datum $u_{0}$ satisfies

$$
a_{0} \leq u_{0} \leq b_{0} \quad \text { a.e in } \Omega
$$

then

$$
a_{0}^{\prime} \leq u_{\lambda} \leq b_{0}^{\prime} \quad \text { a.e in } Q .
$$

Thanks to the convergence (3.9), it is enough to check that

$$
a_{0}^{\prime} \leq u_{\lambda, \tau}^{k} \leq b_{0}^{\prime} \quad \text { a.e. in } \Omega, \quad \text { for } k=0, \ldots, N .
$$

By contradiction, let $k$ be the smallest index such that $u_{\lambda, \tau}^{k}>b_{0}^{\prime}$ on a set of positive measure in $\Omega$. Then, testing the analogue of (3.7) by $\left(u_{\lambda, \tau}^{k}-b_{0}^{\prime}\right)^{+}$we have

$$
\begin{aligned}
\int_{\Omega}(I & \left.+\beta_{\lambda}\right)\left(\frac{u_{\lambda, \tau}^{k}-u_{\lambda, \tau}^{k-1}}{\tau}\right)\left(u_{\lambda, \tau}^{k}-b_{0}^{\prime}\right)^{+}+\int_{\Omega}\left|\nabla\left(u_{\lambda, \tau}^{k}-b_{0}^{\prime}\right)^{+}\right|^{2} \\
& =\int_{\Omega}\left(\mu_{\lambda}-g_{\lambda}-\lambda u_{\lambda, \tau}^{k}-\gamma_{\lambda}\left(u_{\lambda, \tau}^{k}\right)+K T_{\lambda}(I+\lambda \gamma)^{-1}\left(u_{\lambda, \tau}^{k}\right)\right)\left(u_{\lambda, \tau}^{k}-b_{0}^{\prime}\right)^{+} \\
& =\int_{\left\{u_{\lambda, \tau}^{k}>b_{0}^{\prime}\right\}}\left(\mu_{\lambda}-g_{\lambda}-\lambda u_{\lambda, \tau}^{k}-\gamma_{\lambda}\left(u_{\lambda, \tau}^{k}\right)+K T_{\lambda}(I+\lambda \gamma)^{-1}\left(u_{\lambda, \tau}^{k}\right)\right)\left(u_{\lambda, \tau}^{k}-b_{0}^{\prime}\right) .
\end{aligned}
$$


Let us show that the right-hand side of the above equation is non positive if

$$
\lambda<\min \left\{\frac{1}{|\bar{b}|}, \frac{1}{\left|b_{0}^{\prime}\right|}\right\},
$$

which is clearly not restrictive. Indeed, on the set $\left\{u_{\lambda, \tau}^{k}>b_{0}^{\prime}\right\}$, owing to (3.14) we have that $(I+\lambda \gamma)^{-1}\left(u_{\lambda, \tau}^{k}\right) \geq \bar{b}$ and consequently, as $\frac{1}{\lambda}>|\bar{b}|$, also that

$$
T_{\lambda}(I+\lambda \gamma)^{-1}\left(u_{\lambda, \tau}^{k}\right) \leq(I+\lambda \gamma)^{-1}\left(u_{\lambda, \tau}^{k}\right) \text { a.e. in }\left\{u_{\lambda, \tau}^{k}>b_{0}^{\prime}\right\}
$$

Recalling the definition of the Yosida approximation

$$
\gamma_{\lambda}=\frac{I-(I+\lambda \gamma)^{-1}}{\lambda}
$$

we observe that $\gamma_{\lambda}(r)=\gamma\left((I+\lambda \gamma)^{-1}(r)\right)$ for every $r \in \mathbb{R}$. Therefore, by (3.11) we infer that, on the set $\left\{u_{\lambda, \tau}^{k}>b_{0}^{\prime}\right\}$,

$$
\begin{aligned}
& \lambda u_{\lambda, \tau}^{k}+\gamma_{\lambda}\left(u_{\lambda, \tau}^{k}\right)-K T_{\lambda}(I+\lambda \gamma)^{-1}\left(u_{\lambda, \tau}^{k}\right) \\
& =\lambda u_{\lambda, \tau}^{k}+\gamma\left((I+\lambda \gamma)^{-1}\left(u_{\lambda, \tau}^{k}\right)\right)-K(I+\lambda \gamma)^{-1}\left(u_{\lambda, \tau}^{k}\right) \\
& \quad \quad+K(I+\lambda \gamma)^{-1}\left(u_{\lambda, \tau}^{k}\right)-K T_{\lambda}(I+\lambda \gamma)^{-1}\left(u_{\lambda, \tau}^{k}\right) \\
& \quad \geq \lambda u_{\lambda, \tau}^{k}+\psi^{\prime}\left((I+\lambda \gamma)^{-1}\left(u_{\lambda, \tau}^{k}\right)\right) \\
& \geq \lambda b_{0}^{\prime}+M+1 \geq M
\end{aligned}
$$

where we have used that $\lambda<\frac{1}{\left|b_{0}^{\prime}\right|}$.

Hence, recalling (3.10) we deduce that

$$
\int_{\left\{u_{\lambda, \tau}^{k}>b_{0}^{\prime}\right\}}\left(\mu_{\lambda}-g_{\lambda}-\lambda u_{\lambda, \tau}^{k}-\gamma_{\lambda}\left(u_{\lambda, \tau}^{k}\right)+K T_{\lambda}\left(u_{\lambda, \tau}^{k}\right)\right)\left(u_{\lambda, \tau}^{k}-b_{0}^{\prime}\right) \leq 0 .
$$

This implies that

$$
\int_{\left\{u_{\lambda, \tau}^{k}>b_{0}^{\prime}\right\}}\left(I+\beta_{\lambda}\right)\left(\frac{u_{\lambda, \tau}^{k}-u_{\lambda, \tau}^{k-1}}{\tau}\right)\left(u_{\lambda, \tau}^{k}-b_{0}^{\prime}\right) \leq 0 .
$$

Now, on $\left\{u_{\lambda, \tau}^{k}>b_{0}^{\prime}\right\}$ we must have $u_{\lambda, \tau}^{k}>b_{0}^{\prime} \geq u_{\lambda, \tau}^{k-1}$ because of the definition of $k$. Thus, in view of the monotonicity of $\beta_{\lambda}$ and the fact that $\beta_{\lambda}(0)=0$, the integrand in (3.16) is positive. Since $\left\{u_{\lambda, \tau}^{k}>b_{0}^{\prime}\right\}$ has positive measure by assumption this leads to a contradiction.

The above argument implies that the Rothe approximation $u_{\lambda, \tau}^{k}$ satisfies the bound

$$
u_{\lambda, \tau} \leq b_{0}^{\prime} \quad \text { a.e. in } Q
$$

A similar procedure can be used to prove that $u_{\lambda, \tau} \geq a_{0}^{\prime}$ a.e. in $Q$ (for brevity we omit the details), hence (3.15) follows. Consequently, noting also that

$$
a_{0}^{\prime} \leq(I+\lambda \gamma)^{-1}\left(u_{\lambda}\right) \leq b_{0}^{\prime} \quad \text { a.e. in } Q
$$


and $\gamma_{\lambda}\left(u_{\lambda}\right)=\gamma\left((I+\lambda \gamma)^{-1}\left(u_{\lambda}\right)\right)$, since $\gamma \in L^{\infty}\left(a_{0}^{\prime}, b_{0}^{\prime}\right)$ by (2.2) and (2.6), we infer that

$$
\left\|\gamma_{\lambda}\left(u_{\lambda}\right)\right\|_{L^{\infty}(Q)} \leq c .
$$

Taking now the duality pairing between (3.4) and $-\Delta \partial_{t} u_{\lambda}$, integrating by parts we have

$$
\begin{aligned}
& \int_{Q_{t}}\left|\nabla \partial_{t} u_{\lambda}\right|^{2}+\int_{Q_{t}} \beta_{\lambda}^{\prime}\left(\partial_{t} u_{\lambda}\right)\left|\nabla \partial_{t} u_{\lambda}\right|^{2}+\frac{1}{2} \int_{\Omega}\left|\Delta u_{\lambda}(t)\right|^{2}+\frac{\lambda}{2} \int_{\Omega}\left|\nabla u_{\lambda}(t)\right|^{2} \\
& =\frac{1}{2} \int_{\Omega}\left(\left|\Delta u_{0}\right|^{2}+\lambda\left|\nabla u_{0}\right|^{2}\right)+\int_{Q_{t}} \nabla \mu_{\lambda} \cdot \nabla \partial_{t} u_{\lambda} \\
& -\int_{Q_{t}} \partial_{t}\left(g_{\lambda}+\gamma_{\lambda}\left(u_{\lambda}\right)-K T_{\lambda}(I+\lambda \gamma)^{-1}\left(u_{\lambda}\right)\right) \Delta u_{\lambda} \\
& +\int_{\Omega}\left(g_{\lambda}+\gamma_{\lambda}\left(u_{\lambda}\right)-K T_{\lambda}(I+\lambda \gamma)^{-1}\left(u_{\lambda}\right)\right)(t) \Delta u_{\lambda}(t) \\
& -\int_{\Omega}\left(g_{\lambda}(0)+\gamma_{\lambda}\left(u_{0}\right)-K T_{\lambda}(I+\lambda \gamma)^{-1}\left(u_{0}\right)\right) \Delta u_{0} .
\end{aligned}
$$

The first two terms on the right-hand side can be treated by the assumptions on $u_{0}$ and the Young inequality. About the third term, note that, since $\gamma^{\prime} \in C^{0}\left(\left[a_{0}^{\prime}, b_{0}^{\prime}\right]\right)$ by (2.2), from (3.17) it follows that

$$
\left|\partial_{t} \gamma_{\lambda}\left(u_{\lambda}\right)\right|=\left|\gamma_{\lambda}^{\prime}\left(u_{\lambda}\right) \partial_{t} u_{\lambda}\right| \leq \gamma^{\prime}\left((I+\lambda \gamma)^{-1}\left(u_{\lambda}\right)\right)\left|\partial_{t} u_{\lambda}\right| \leq c\left|\partial_{t} u_{\lambda}\right| .
$$

Hence, using the estimates (3.6) and (3.10), as well as the properties of $\left(g_{\lambda}\right)_{\lambda}$, again by the Young inequality we infer that

$$
\begin{aligned}
\frac{1}{2} \int_{Q_{t}}\left|\nabla \partial_{t} u_{\lambda}\right|^{2} & +\int_{Q_{t}} \beta_{\lambda}^{\prime}\left(\partial_{t} u_{\lambda}\right)\left|\nabla \partial_{t} u_{\lambda}\right|^{2}+\frac{1}{4} \int_{\Omega}\left|\Delta u_{\lambda}(t)\right|^{2}+\frac{\lambda}{2} \int_{\Omega}\left|\nabla u_{\lambda}(t)\right|^{2} \\
& \leq c\left(1+\int_{Q_{t}}\left|\Delta u_{\lambda}\right|^{2}\right) .
\end{aligned}
$$

The Gronwall lemma yields then

$$
\left\|\Delta u_{\lambda}\right\|_{L^{\infty}(0, T ; H)} \leq c,
$$

whence, by comparison in (3.4), we also have

$$
\left\|\beta_{\lambda}\left(\partial_{t} u_{\lambda}\right)\right\|_{L^{\infty}(0, T ; H)} \leq c .
$$

Proceeding now as in [5, §6], we can conclude.

\subsection{The continuous dependence result}

We focus here on the proof of Theorem 2.3. Let $\left(u_{i}, \mu_{i}, \xi_{i}\right)$ satisfy (2.11)-(2.18) with respect to the data $\left(u_{0, i}, g_{i}\right)$, for $i=1,2$ : then, setting $u:=u_{1}-u_{2}, \mu:=\mu_{1}-\mu_{2}$, $\xi:=\xi_{1}-\xi_{2} u_{0}:=u_{0,1}-u_{0,2}$, and $g:=g_{1}-g_{2}$, we have

$$
\begin{aligned}
\partial_{t} u-\Delta \mu=0 & \text { in } Q, \\
\mu=\varepsilon \partial_{t} u+\xi-\delta \Delta u+\psi^{\prime}\left(u_{1}\right)-\psi^{\prime}\left(u_{2}\right)+g & \text { in } Q, \\
u(0)=u_{0} & \text { in } \Omega .
\end{aligned}
$$


Testing the first equation by $\mu$, the second by $\partial_{t} u$ and taking the difference we deduce, by monotonicity of $\beta$, for all $t \in[0, T]$,

$$
\begin{aligned}
& \int_{Q_{t}}|\nabla \mu|^{2}+\varepsilon \int_{Q_{t}}\left|\partial_{t} u\right|^{2}+\int_{Q_{t}} \xi \partial_{t} u+\frac{\delta}{2} \int_{\Omega}|\nabla u(t)|^{2} \\
& \leq \frac{\delta}{2}\left\|\nabla u_{0}\right\|_{H}^{2}-\int_{Q_{t}}\left(\psi^{\prime}\left(u_{1}\right)-\psi^{\prime}\left(u_{2}\right)\right) \partial_{t} u-\int_{Q_{t}} g \partial_{t} u .
\end{aligned}
$$

Now, the fact that $u_{1}, u_{2} \in\left[a_{0}^{\prime}, b_{0}^{\prime}\right] \subset(a, b)$ for some $a_{0}^{\prime}, b_{0}^{\prime}$ yields

$$
\left|\psi^{\prime}\left(u_{1}\right)-\psi^{\prime}\left(u_{2}\right)\right| \leq\left\|\psi^{\prime \prime}\right\|_{C^{0}\left(\left[a_{0}^{\prime}, b_{0}^{\prime}\right]\right)}|u| .
$$

Hence, using the Young inequality and the fact that

$$
u(t)=u_{0}+\int_{0}^{t} \partial_{t} u(s) d s,
$$

we are left with

$$
\begin{aligned}
& \int_{Q_{t}}|\nabla \mu|^{2}+\frac{\varepsilon}{2} \int_{Q_{t}}\left|\partial_{t} u\right|^{2}+\int_{Q_{t}} \xi \partial_{t} u+\frac{\delta}{2} \int_{\Omega}|\nabla u(t)|^{2} \\
& \leq \frac{\delta}{2}\left\|\nabla u_{0}\right\|_{H}^{2}+\frac{1}{\varepsilon}\left\|\psi^{\prime \prime}\right\|_{C^{0}\left(\left[a_{0}^{\prime}, b_{0}^{\prime}\right]\right)}^{2} \int_{Q_{t}}|u|^{2}+\frac{1}{\varepsilon}\|g\|_{L^{2}(0, T ; H)}^{2} \\
& \leq \frac{\delta}{2}\left\|\nabla u_{0}\right\|_{H}^{2}+\frac{2 T}{\varepsilon}\left\|u_{0}\right\|_{H}^{2}+\frac{2 T}{\varepsilon}\left\|\psi^{\prime \prime}\right\|_{C^{0}\left(\left[a_{0}^{\prime}, b_{0}^{\prime}\right]\right)}^{2} \int_{0}^{t} \int_{Q_{s}}\left|\partial_{t} u\right|^{2} d s+\frac{1}{\varepsilon}\|g\|_{L^{2}(0, T ; H)}^{2} .
\end{aligned}
$$

The Gronwall lemma yields then the desired continuous dependence estimate (2.19).

\section{Proof of Theorem 2.4}

This section is devoted to the proof of Theorem 2.4. Since $\delta>0$ is fixed and we let $\varepsilon \searrow 0$, in order to avoid heavy notations we will not write explicitly the dependence on $\delta$ for the quantities in play. In particular, let $\left(u_{\varepsilon}, \mu_{\varepsilon}, \xi_{\varepsilon}\right)$ be any solution satisfying (2.11)-(2.18) for every $\varepsilon>0$.

\subsection{First estimate}

We test (2.16) by $\mu_{\varepsilon}$, (2.17) by $\partial_{t} u_{\varepsilon}$ and subtract, obtaining

$$
\begin{aligned}
\int_{Q_{t}}\left|\nabla \mu_{\varepsilon}\right|^{2} & +\varepsilon \int_{Q_{t}}\left|\partial_{t} u_{\varepsilon}\right|^{2}+\int_{Q_{t}} \xi_{\varepsilon} \partial_{t} u_{\varepsilon}+\frac{\delta}{2} \int_{\Omega}\left|\nabla u_{\varepsilon}(t)\right|^{2}+\int_{\Omega} \psi\left(u_{\varepsilon}(t)\right) \\
& =\frac{\delta}{2} \int_{\Omega}\left|\nabla u_{0}\right|^{2}+\int_{\Omega} \psi\left(u_{0}\right)-\int_{Q_{t}} g_{\varepsilon} \partial_{t} u_{\varepsilon} \quad \forall t \in[0, T] .
\end{aligned}
$$

Now, by (2.6) $-(2.7)$ we have

$$
\psi\left(u_{0}\right)=\widehat{\gamma}\left(u_{0}\right)-\frac{K}{2}\left|u_{0}\right|^{2}+\psi\left(r_{0}\right)+\frac{K}{2}\left|r_{0}\right|^{2},
$$


where, recalling that $\psi^{\prime}\left(u_{0}\right) \in H$ by (2.20), hence also $\gamma\left(u_{0}\right) \in H$,

$$
\widehat{\gamma}\left(u_{0}\right) \leq \widehat{\gamma}\left(u_{0}\right)+\widehat{\gamma^{-1}}\left(\gamma\left(u_{0}\right)\right)=\gamma\left(u_{0}\right) u_{0} \in L^{1}(\Omega) .
$$

Therefore, we see that $\psi\left(u_{0}\right) \in L^{1}(\Omega)$. By the monotonicity of $\beta$ and conditions (2.20), (2.21) and (2.24), integrating by parts in time the last term we infer that there exists $c>0$, independent of $\varepsilon$, such that

$$
\begin{aligned}
& \int_{Q_{t}}\left|\nabla \mu_{\varepsilon}\right|^{2}+\varepsilon \int_{Q_{t}}\left|\partial_{t} u_{\varepsilon}\right|^{2}+\frac{\delta}{2} \int_{\Omega}\left|\nabla u_{\varepsilon}(t)\right|^{2}+C_{1} \int_{\Omega}\left|u_{\varepsilon}(t)\right|^{2} \\
& \leq c+\int_{Q_{t}} \partial_{t} g_{\varepsilon} u_{\varepsilon}-\int_{\Omega} g_{\varepsilon}(t) u_{\varepsilon}(t)+\int_{\Omega} g(0) u_{0} \\
& \leq c+\int_{Q_{t}}\left|u_{\varepsilon}\right|^{2}+\frac{1}{4} \int_{Q}\left|\partial_{t} g_{\varepsilon}\right|^{2}+\frac{C_{1}}{2} \int_{\Omega}\left|u_{\varepsilon}(t)\right|^{2}+\frac{1}{2 C_{1}}\left\|g_{\varepsilon}(t)\right\|_{H}^{2}+\|g(0)\|_{H}\left\|u_{0}\right\|_{H} .
\end{aligned}
$$

Rearranging the terms and recalling that $\left(g_{\varepsilon}\right)_{\varepsilon}$ is bounded in $H^{1}(0, T ; H)$ independently of $\varepsilon$ by (2.24), an application of the Gronwall lemma leads to

$$
\left\|\mu_{\varepsilon}\right\|_{L^{2}\left(0, T ; V_{0}\right)}+\left\|u_{\varepsilon}\right\|_{L^{\infty}(0, T ; V)}+\varepsilon^{1 / 2}\left\|\partial_{t} u_{\varepsilon}\right\|_{L^{2}(0, T ; H)} \leq c
$$

and by comparison in (2.16) we also deduce that

$$
\left\|\partial_{t} u_{\varepsilon}\right\|_{L^{2}\left(0, T ; V_{0}^{*}\right)} \leq c .
$$

\subsection{Second estimate}

In order to derive this estimate first we need to identify the initial values of the solutions $\mu_{0 \varepsilon}:=\mu_{\varepsilon}(0)$ and $u_{0 \varepsilon}^{\prime}:=\partial_{t} u_{\varepsilon}(0)$.

Lemma 4.1. For every $\varepsilon>0$, there exists a unique triplet $\left(\mu_{0 \varepsilon}, u_{0 \varepsilon}^{\prime}, \xi_{0 \varepsilon}\right) \in W_{0} \times H \times H$ such that

$$
u_{0 \varepsilon}^{\prime}-\Delta \mu_{0 \varepsilon}=0, \quad \mu_{0 \varepsilon}=\varepsilon u_{0 \varepsilon}^{\prime}+\xi_{0 \varepsilon}-\delta \Delta u_{0}+\psi^{\prime}\left(u_{0}\right)+g(0), \quad \xi_{0 \varepsilon} \in \beta\left(u_{0 \varepsilon}^{\prime}\right)
$$

almost everywhere in $\Omega$. Moreover, there exists a positive constant $c$, independent of $\varepsilon$, such that

$$
\int_{\Omega}\left|\nabla \mu_{0 \varepsilon}\right|^{2}+\varepsilon \int_{\Omega}\left|u_{0 \varepsilon}^{\prime}\right|^{2}+\int_{\Omega} \widehat{\beta^{-1}}\left(\xi_{0 \varepsilon}\right) \leq c \quad \forall \varepsilon>0 .
$$

Proof. Since $z_{0}:=-\delta \Delta u_{0}+\psi^{\prime}\left(u_{0}\right)+g(0) \in H$, existence and uniqueness of $\left(\mu_{0 \varepsilon}, u_{0 \varepsilon}^{\prime}, \xi_{0 \varepsilon}\right)$ follows from the maximal monotonicity of $\beta$, arguing as in [5, p. 1006]. Moreover, testing the first equation by $\mu_{0 \varepsilon}$, the second by $u_{0 \varepsilon}^{\prime}$ and taking the difference we have

$$
\int_{\Omega}\left|\nabla \mu_{0 \varepsilon}\right|^{2}+\varepsilon \int_{\Omega}\left|u_{0 \varepsilon}^{\prime}\right|^{2}+\int_{\Omega} \xi_{0 \varepsilon} u_{0 \varepsilon}^{\prime}+\int_{\Omega} z_{0} u_{0 \varepsilon}^{\prime}=0 .
$$

Since $\xi_{0 \varepsilon} \in \beta\left(u_{0 \varepsilon}^{\prime}\right)$, on the left-hand side we have that $\xi_{0 \varepsilon} u_{0 \varepsilon}^{\prime}=\widehat{\beta}\left(u_{0 \varepsilon}^{\prime}\right)+\widehat{\beta^{-1}}\left(\xi_{0 \varepsilon}\right)$. Moreover, by the Young inequality we have

$$
\begin{gathered}
\int_{\Omega}\left|\nabla \mu_{0 \varepsilon}\right|^{2}+\varepsilon \int_{\Omega}\left|u_{0 \varepsilon}^{\prime}\right|^{2}+\int_{\Omega} \widehat{\beta}\left(u_{0 \varepsilon}^{\prime}\right)+\int_{\Omega} \widehat{\beta^{-1}}\left(\xi_{0 \varepsilon}\right) \\
=-\int_{\Omega} z_{0} u_{0 \varepsilon}^{\prime} \leq \int_{\Omega} \widehat{\beta^{-1}}\left(-z_{0}\right)+\int_{\Omega} \widehat{\beta}\left(u_{0 \varepsilon}^{\prime}\right)
\end{gathered}
$$

from which the estimate follows thanks to hypothesis (2.22). 
Now, we proceed formally, testing (2.16) by $\partial_{t} \mu_{\varepsilon}$, the time-derivative of (2.17) by $\partial_{t} u_{\varepsilon}$ and subtracting: a rigorous computation can be obtained through a discretization in time (for further details, see for example [5, § 5.2]). We obtain then, recalling the previous lemma and that $\psi^{\prime \prime} \geq-K$ by (2.5),

$$
\begin{aligned}
& \frac{1}{2} \int_{\Omega}\left|\nabla \mu_{\varepsilon}(t)\right|^{2}+\frac{\varepsilon}{2} \int_{\Omega}\left|\partial_{t} u_{\varepsilon}(t)\right|^{2}+\int_{\Omega} \widehat{\beta^{-1}}\left(\xi_{\varepsilon}(t)\right)+\delta \int_{Q_{t}}\left|\nabla \partial_{t} u_{\varepsilon}\right|^{2} \\
& =\frac{1}{2} \int_{\Omega}\left|\nabla \mu_{0 \varepsilon}\right|^{2}+\frac{\varepsilon}{2} \int_{\Omega}\left|u_{0 \varepsilon}^{\prime}\right|^{2}+\int_{\Omega} \widehat{\beta^{-1}}\left(\xi_{0 \varepsilon}\right)-\int_{Q_{t}}\left(\partial_{t} g_{\varepsilon}+\psi^{\prime \prime}\left(u_{\varepsilon}\right) \partial_{t} u_{\varepsilon}\right) \partial_{t} u_{\varepsilon} \\
& \leq c+\frac{1}{4}\left\|\partial_{t} g_{\varepsilon}\right\|_{L^{2}(0, T ; H)}^{2}+(1+K) \int_{Q_{t}}\left|\partial_{t} u_{\varepsilon}\right|^{2} .
\end{aligned}
$$

By the compactness inequality (2.1), we can handle the last term on the right-hand side as

$$
(1+K) \int_{Q_{t}}\left|\partial_{t} u_{\varepsilon}\right|^{2} \leq \frac{\delta}{2} \int_{Q_{t}}\left|\nabla \partial_{t} u_{\varepsilon}\right|^{2}+c\left\|\partial_{t} u_{\varepsilon}\right\|_{L^{2}\left(0, T ; V_{0}^{*}\right)}^{2},
$$

so that by (4.2) and again (2.1) we infer (possibly renominating $c$ ) that

$$
\left\|\mu_{\varepsilon}\right\|_{L^{\infty}\left(0, T ; V_{0}\right)}+\left\|\partial_{t} u_{\varepsilon}\right\|_{L^{2}(0, T ; V)}+\varepsilon^{1 / 2}\left\|\partial_{t} u_{\varepsilon}\right\|_{L^{\infty}(0, T ; H)} \leq c
$$

and, by comparison in (2.16), also

$$
\left\|\partial_{t} u_{\varepsilon}\right\|_{L^{\infty}\left(0, T ; V_{0}^{*}\right)}+\left\|\mu_{\varepsilon}\right\|_{L^{2}\left(0, T ; H^{3}(\Omega)\right)} \leq c .
$$

\subsection{Third estimate under assumption (2.23)}

We test (2.17) by $-\delta \Delta \partial_{t} u_{\varepsilon}+\partial_{t} \gamma\left(u_{\varepsilon}\right)$ : to this end, note that since $\partial_{t} u_{\varepsilon} \in L^{2}(0, T ; V)$ only, then $-\Delta \partial_{t} u_{\varepsilon}$ has to be interpreted as an element in $L^{2}\left(0, T ; V^{*}\right)$. However, be aware that $\xi_{\varepsilon} \in L^{\infty}(0, T ; H)$, so that the estimate that we perform is formal. To be rigorous, one should regularize $\beta$ with its Yosida approximation $\beta_{\lambda}$ and then carry out the computations: as a matter of fact, it is readily seen that the resulting estimate would be independent of $\lambda$, so that we avoid such technicalities here. We have

$$
\begin{aligned}
& \frac{1}{2} \int_{\Omega}\left|-\delta \Delta u_{\varepsilon}+\gamma\left(u_{\varepsilon}\right)\right|^{2}(t) \\
& \quad+\varepsilon \delta \int_{Q_{t}}\left|\nabla \partial_{t} u_{\varepsilon}\right|^{2}+\delta \int_{Q_{t}} \nabla \xi_{\varepsilon} \cdot \nabla \partial_{t} u_{\varepsilon}+\varepsilon \int_{Q_{t}} \gamma^{\prime}\left(u_{\varepsilon}\right)\left|\partial_{t} u_{\varepsilon}\right|^{2}+\int_{Q_{t}} \gamma^{\prime}\left(u_{\varepsilon}\right) \xi_{\varepsilon} \partial_{t} u_{\varepsilon} \\
& =\frac{1}{2} \int_{\Omega}\left|-\delta \Delta u_{0}+\gamma\left(u_{0}\right)\right|^{2}+\delta \int_{Q_{t}} \nabla \mu_{\varepsilon} \cdot \nabla \partial_{t} u_{\varepsilon}+\int_{Q_{t}} \mu_{\varepsilon} \gamma^{\prime}\left(u_{\varepsilon}\right) \partial_{t} u_{\varepsilon} \\
& \quad+\int_{Q_{t}}\left(\partial_{t} g_{\varepsilon}-K \partial_{t} u_{\varepsilon}\right)\left(-\delta \Delta u_{\varepsilon}+\gamma\left(u_{\varepsilon}\right)\right)-\int_{\Omega}\left(g_{\varepsilon}(t)-K u_{\varepsilon}(t)\right)\left(-\delta \Delta u_{\varepsilon}+\gamma\left(u_{\varepsilon}\right)\right)(t) \\
& \quad+\int_{\Omega}\left(g(0)-K u_{0}\right)\left(-\delta \Delta u_{0}+\gamma\left(u_{0}\right)\right) \quad \forall t \in[0, T] .
\end{aligned}
$$

Now, as we have anticipated, if we replace $\beta$ with its Yosida approximation $\beta_{\lambda}$, the third term on the left-hand side would give the contribution

$$
\int_{Q_{t}} \beta_{\lambda}^{\prime}\left(\partial_{t} u_{\varepsilon, \lambda}\right)\left|\nabla \partial_{t} u_{\varepsilon, \lambda}\right|^{2} \geq 0 \quad \forall \lambda>0 .
$$


Moreover, it is also clear by the properties of $\beta$ that the last term on the left-hand side is nonnegative. On the right hand side, the first term is finite by assumption (2.20) while the second term is bounded uniformly in $\varepsilon$ by (4.3). Furthermore, by (4.1), (4.3) -(4.4) and (2.23), using the continuous embeddings $V \hookrightarrow L^{6}(\Omega)$ and $H^{3}(\Omega) \hookrightarrow L^{\infty}(\Omega)$ we have

$$
\begin{aligned}
\int_{Q} \mu_{\varepsilon} \gamma^{\prime}\left(u_{\varepsilon}\right) \partial_{t} u_{\varepsilon} & \leq \int_{0}^{T}\left\|\mu_{\varepsilon}(t)\right\|_{L^{\infty}(\Omega)}\left\|\gamma^{\prime}\left(u_{\varepsilon}(t)\right)\right\|_{L^{6 / 5}(\Omega)}\left\|\partial_{t} u_{\varepsilon}(t)\right\|_{L^{6}(\Omega)} d t \\
& \leq c\left\|\mu_{\varepsilon}\right\|_{L^{2}\left(0, T ; H^{3}(\Omega)\right)}\left\|\partial_{t} u_{\varepsilon}\right\|_{L^{2}(0, T ; V)}\left\|\gamma^{\prime}\left(u_{\varepsilon}\right)\right\|_{L^{\infty}\left(0, T ; L^{6 / 5}(\Omega)\right)} \\
& \leq c\left(1+\left\|u_{\varepsilon}\right\|_{L^{\infty}\left(0, T ; L^{6}(\Omega)\right)}^{5}\right) \leq c
\end{aligned}
$$

for a certain constant $c>0$ that we have updated step by step. Finally, we handle the last three terms on the right-hand side using Young's inequality, the estimate (4.1) and the assumptions (2.20) and (2.24) by

$$
c+\int_{Q_{t}}\left|-\delta \Delta u_{\varepsilon}+\gamma\left(u_{\varepsilon}\right)\right|^{2}+\frac{1}{4} \int_{\Omega}\left|-\delta \Delta u_{\varepsilon}+\gamma\left(u_{\varepsilon}\right)\right|^{2}(t) .
$$

Consequently, rearranging the terms and using the Gronwall inequality lead to

$$
\left\|-\delta \Delta u_{\varepsilon}+\gamma\left(u_{\varepsilon}\right)\right\|_{L^{\infty}(0, T ; H)} \leq c .
$$

Since $\gamma$ is monotone, testing $-\delta \Delta u_{\varepsilon}+\gamma\left(u_{\varepsilon}\right)$ by $-\Delta u_{\varepsilon}$, integrating by parts and using the Young inequality yield (recall that $\delta>0$ is fixed here)

$$
\begin{aligned}
\delta \int_{\Omega}\left|\Delta u_{\varepsilon}\right|^{2} & \leq \delta \int_{\Omega}\left|\Delta u_{\varepsilon}\right|^{2}+\int_{\Omega} \gamma^{\prime}\left(u_{\varepsilon}\right)\left|\nabla u_{\varepsilon}\right|^{2}=\int_{\Omega}\left(-\Delta u_{\varepsilon}\right)\left(-\delta \Delta u_{\varepsilon}+\gamma\left(u_{\varepsilon}\right)\right) \\
& \leq \frac{\delta}{2} \int_{\Omega}\left|\Delta u_{\varepsilon}\right|^{2}+\frac{1}{2 \delta} \int_{\Omega}\left|-\delta \Delta u_{\varepsilon}+\gamma\left(u_{\varepsilon}\right)\right|^{2}
\end{aligned}
$$

almost everywhere in $(0, T)$. Rearranging the terms and invoking elliptic regularity we deduce then

$$
\left\|u_{\varepsilon}\right\|_{L^{\infty}\left(0, T ; W_{\mathbf{n}}\right)}+\left\|\gamma\left(u_{\varepsilon}\right)\right\|_{L^{\infty}(0, T ; H)} \leq c
$$

and consequently, by comparison in (2.17),

$$
\left\|\xi_{\varepsilon}\right\|_{L^{\infty}(0, T ; H)} \leq c
$$

\subsection{Third estimate under assumption (2.36)}

By (2.36) and (4.3) we immediately have

$$
\left\|\xi_{\varepsilon}\right\|_{L^{2}(0, T ; H)} \leq c .
$$

Then, with the help of a comparison in (2.17) we see that

$$
\left\|-\delta \Delta u_{\varepsilon}+\gamma\left(u_{\varepsilon}\right)\right\|_{L^{2}(0, T ; H)} \leq c .
$$

Hence, by applying the same argument leading to (4.5) we arrive at

$$
\left\|u_{\varepsilon}\right\|_{L^{2}\left(0, T ; W_{\mathbf{n}}\right)}+\left\|\gamma\left(u_{\varepsilon}\right)\right\|_{L^{2}(0, T ; H)} \leq c .
$$




\subsection{Passage to the limit}

Let us assume first (2.23) . Then, by the estimates (4.1)-(4.6) we deduce that there is a triplet $(u, \mu, \xi)$ such that

$$
\begin{gathered}
u \in W^{1, \infty}\left(0, T ; V_{0}^{*}\right) \cap H^{1}(0, T ; V) \cap L^{\infty}\left(0, T ; W_{\mathbf{n}}\right), \\
\mu \in L^{\infty}\left(0, T ; V_{0}\right) \cap L^{2}\left(0, T ; H^{3}(\Omega)\right), \quad \xi \in L^{\infty}(0, T ; H), \quad \eta \in L^{\infty}(0, T ; H)
\end{gathered}
$$

and, along a subsequence that we still denote by $\varepsilon$ for simplicity,

$$
\begin{array}{r}
u_{\varepsilon} \stackrel{*}{\rightarrow} u \quad \text { in } W^{1, \infty}\left(0, T ; V_{0}^{*}\right) \cap L^{\infty}\left(0, T ; W_{\mathbf{n}}\right), \quad u_{\varepsilon} \rightarrow u \quad \text { in } H^{1}(0, T ; V), \\
\mu_{\varepsilon} \stackrel{*}{\rightarrow} \mu \quad \text { in } L^{\infty}\left(0, T ; V_{0}\right), \quad \mu_{\varepsilon} \rightarrow \mu \quad \text { in } L^{2}\left(0, T ; H^{3}(\Omega)\right), \\
\xi_{\varepsilon} \stackrel{*}{\rightarrow} \xi \quad \text { in } L^{\infty}(0, T ; H), \quad \gamma\left(u_{\varepsilon}\right) \stackrel{*}{\rightarrow} \eta \quad \text { in } L^{\infty}(0, T ; H), \\
\varepsilon \partial_{t} u_{\varepsilon} \rightarrow 0 \quad \text { in } L^{\infty}(0, T ; H) .
\end{array}
$$

Now, from the first two convergences and a classical compactness result (see e.g. [31, Cor. 4, p. 85]), we have that

$$
u_{\varepsilon} \rightarrow u \quad \text { in } C^{0}([0, T] ; V),
$$

which immediately implies that $\eta=\gamma(u)=\psi^{\prime}(u)+K u$ a.e. in $Q$ by the strong-weak closure (see, e.g., [3, Prop. 2.1, p. 29]) of the maximal monotone operator $\gamma$.

Moreover, letting then $\varepsilon \searrow 0$ in (2.16) $-(2.17)$, we infer by the weak convergences that (2.29) - (2.30) hold. Now, proceeding as in the first estimate we have that

$$
\begin{aligned}
\int_{Q} \xi_{\varepsilon} \partial_{t} u_{\varepsilon} \leq & \frac{\delta}{2} \int_{\Omega}\left|\nabla u_{0}\right|^{2}+\int_{\Omega} \widehat{\gamma}\left(u_{0}\right)-\int_{Q} g_{\varepsilon} \partial_{t} u_{\varepsilon}+K \int_{Q} u_{\varepsilon} \partial_{t} u_{\varepsilon} \\
& -\int_{Q}\left|\nabla \mu_{\varepsilon}\right|^{2}-\frac{\delta}{2} \int_{\Omega}\left|\nabla u_{\varepsilon}(T)\right|^{2}-\int_{\Omega} \widehat{\gamma}\left(u_{\varepsilon}(T)\right)
\end{aligned}
$$

so that, using the convergences already proved and the (weak) lower semicontinuity of the convex integrands, we infer that

$$
\begin{aligned}
\limsup _{\varepsilon \searrow 0} \int_{Q} \xi_{\varepsilon} \partial_{t} u_{\varepsilon} \leq & \frac{\delta}{2} \int_{\Omega}\left|\nabla u_{0}\right|^{2}+\int_{\Omega} \widehat{\gamma}\left(u_{0}\right)-\int_{Q} g \partial_{t} u+K \int_{Q} u \partial_{t} u \\
& -\int_{Q}|\nabla \mu|^{2}-\frac{\delta}{2} \int_{\Omega}|\nabla u(T)|^{2}-\int_{\Omega} \widehat{\gamma}(u(T)) .
\end{aligned}
$$

Since we have already proved (2.29) $-(2.30)$, performing the same computation on the limit problem yields that the right-hand side is exactly $\int_{Q} \xi \partial_{t} u$. Hence,

$$
\limsup _{\varepsilon \searrow 0} \int_{Q} \xi_{\varepsilon} \partial_{t} u_{\varepsilon} \leq \int_{Q} \xi \partial_{t} u,
$$

and this is enough to conclude that $\xi \in \beta\left(\partial_{t} u\right)$ a.e. in $Q$.

If (2.36) is in order, we can proceed in exactly the same way using the estimates (4.7)(4.8) instead of (4.5)-(4.6): note that in this case, by [31, Cor. 4, p. 85] we can only infer the strong convergence

$$
u_{\varepsilon} \rightarrow u \quad \text { in } C^{0}([0, T] ; H) \cap L^{2}(0, T ; V) .
$$

Since also $u_{\varepsilon}(T) \rightarrow u(T)$ in $V$, the argument performed above still works by weak lower semicontinuity. 


\section{Proof of Theorem 2.6}

This section is devoted to the proof of Theorem 2.6. We shall consider $\varepsilon>0$ fixed and we will not write explicitly the dependence on $\varepsilon$ for the quantities in play. Thus, in what follows we shall let $\left(u_{\delta}, \mu_{\delta}, \xi_{\delta}\right)$ be the solution to (2.11) -(2.18) with respect to the data $\left(u_{0 \delta}, g_{\delta}\right)$ for every $\delta>0$.

\subsection{First estimate}

To obtain the first estimate, proceed as in Section 4: we test (2.16) by $\mu_{\delta}$ and we subtract (2.17) tested by $\partial_{t} u_{\delta}$. By integration over $(0, t)$ for $t \in[0, T]$, we obtain

$$
\begin{aligned}
\int_{Q_{t}}\left|\nabla \mu_{\delta}\right|^{2} & +\varepsilon \int_{Q_{t}}\left|\partial_{t} u_{\delta}\right|^{2}+\int_{Q_{t}} \xi_{\delta} \partial_{t} u_{\delta}+\frac{\delta}{2} \int_{\Omega}\left|\nabla u_{\delta}(t)\right|^{2}+\int_{\Omega} \psi\left(u_{\delta}(t)\right) \\
& =\frac{\delta}{2} \int_{\Omega}\left|\nabla u_{0 \delta}\right|^{2}+\int_{\Omega} \psi\left(u_{0 \delta}\right)-\int_{Q_{t}} g_{\delta} \partial_{t} u_{\delta}
\end{aligned}
$$

Since $\psi^{\prime}\left(u_{0 \delta}\right)$ is bounded in $H$ by (2.40), hence also $\psi\left(u_{0 \delta}\right)$ is bounded in $L^{1}(\Omega)$ as already pointed out at the beginning of Section 4.1. Then, the first two terms on the right-hand side are bounded uniformly in $\delta$. Moreover, one has

$$
\int_{Q_{t}} g_{\delta} \partial_{t} u_{\delta} \leq \frac{1}{2 \varepsilon}\left\|g_{\delta}\right\|_{L^{2}(0, T ; H)}^{2}+\frac{\varepsilon}{2} \int_{Q_{t}}\left|\partial_{t} u_{\delta}\right|^{2}
$$

Consequently, recalling also that

$$
\widehat{\beta}\left(\partial_{t} u_{\delta}\right) \leq \widehat{\beta}\left(\partial_{t} u_{\delta}\right)+\widehat{\beta^{-1}}\left(\xi_{\delta}\right)=\xi_{\delta} \partial_{t} u_{\delta},
$$

by the assumption (2.41) on $\left(g_{\delta}\right)_{\delta}$ and the Gronwall lemma we deduce that

$$
\left\|\mu_{\delta}\right\|_{L^{2}\left(0, T ; V_{0}\right)}+\left\|\partial_{t} u_{\delta}\right\|_{L^{2}(0, T ; H)}+\delta^{1 / 2}\left\|u_{\delta}\right\|_{L^{\infty}(0, T ; V)}+\left\|\widehat{\beta}\left(\partial_{t} u_{\delta}\right)\right\|_{L^{1}(Q)} \leq c,
$$

where $c$ is a positive constant independent of $\delta$.

\subsection{Second estimate}

We repeat the same estimate as in Section 4.2. First of all, we need to identify and estimate the initial values of the solutions $\mu_{0 \delta}:=\mu_{\delta}(0)$ and $u_{0 \delta}^{\prime}:=\partial_{t} u_{\delta}(0)$.

Lemma 5.1. For every $\delta>0$, there exists a unique triplet $\left(\mu_{0 \delta}, u_{0 \delta}^{\prime}, \xi_{0 \delta}\right) \in W_{0} \times H \times H$ such that

$$
u_{0 \delta}^{\prime}-\Delta \mu_{0 \delta}=0, \quad \mu_{0 \delta}=\varepsilon u_{0 \delta}^{\prime}+\xi_{0 \delta}-\delta \Delta u_{0 \delta}+\psi^{\prime}\left(u_{0 \delta}\right)+g_{\delta}(0), \quad \xi_{0 \delta} \in \beta\left(u_{0 \delta}^{\prime}\right)
$$

almost everywhere in $\Omega$. Moreover, there exists a positive constant $c$, independent of $\delta$, such that

$$
\int_{\Omega}\left|\nabla \mu_{0 \delta}\right|^{2}+\varepsilon \int_{\Omega}\left|u_{0 \delta}^{\prime}\right|^{2}+\int_{\Omega} \widehat{\beta^{-1}}\left(\xi_{0 \delta}\right) \leq c \quad \forall \delta>0 .
$$


Proof. Since $z_{0 \delta}:=-\delta \Delta u_{0 \delta}+\psi^{\prime}\left(u_{0 \delta}\right)+g_{\delta}(0) \in H$, the existence and uniqueness of $\left(\mu_{0 \delta}, u_{0 \delta}^{\prime}, \xi_{0 \delta}\right)$ follows from the maximal monotonicity of $\beta$, arguing as in Section 4.2. Moreover, testing the first equation by $\mu_{0 \delta}$, the second by $u_{0 \delta}^{\prime}$ and taking the difference we have

$$
\int_{\Omega}\left|\nabla \mu_{0 \delta}\right|^{2}+\varepsilon \int_{\Omega}\left|u_{0 \delta}^{\prime}\right|^{2}+\int_{\Omega} \xi_{0 \delta} u_{0 \delta}^{\prime}+\int_{\Omega} z_{0 \delta} u_{0 \delta}^{\prime}=0 .
$$

By monotonicity of $\beta$, the fact that $z_{0 \delta}$ is bounded in $H$ thanks to the assumptions (2.40)-(2.41), and the Young inequality we have

$$
\int_{\Omega}\left|\nabla \mu_{0 \delta}\right|^{2}+\frac{\varepsilon}{2} \int_{\Omega}\left|u_{0 \delta}^{\prime}\right|^{2}+\int_{\Omega} \widehat{\beta}\left(u_{0 \delta}^{\prime}\right)+\int_{\Omega} \widehat{\beta^{-1}}\left(\xi_{0 \delta}\right)=-\int_{\Omega} z_{0} u_{0 \delta}^{\prime} \leq \frac{1}{2 \varepsilon}\left\|z_{0 \delta}\right\|_{H}^{2} \leq c,
$$

from which the estimate follows.

Performing then the same computations as in Section 4.2 we deduce that

$$
\begin{aligned}
& \frac{1}{2} \int_{\Omega}\left|\nabla \mu_{\delta}(t)\right|^{2}+\frac{\varepsilon}{2} \int_{\Omega}\left|\partial_{t} u_{\delta}(t)\right|^{2}+\int_{\Omega} \widehat{\beta^{-1}}\left(\xi_{\delta}(t)\right)+\delta \int_{Q_{t}}\left|\nabla \partial_{t} u_{\delta}\right|^{2}+\int_{Q_{t}} \gamma^{\prime}\left(u_{\delta}\right)\left|\partial_{t} u_{\delta}\right|^{2} \\
& =\frac{1}{2} \int_{\Omega}\left|\nabla \mu_{0 \delta}\right|^{2}+\frac{\varepsilon}{2} \int_{\Omega}\left|u_{0 \delta}^{\prime}\right|^{2}+\int_{\Omega} \widehat{\beta^{-1}}\left(\xi_{0 \delta}\right)-\int_{Q_{t}}\left(\partial_{t} g_{\delta}-K \partial_{t} u_{\delta}\right) \partial_{t} u_{\delta} \\
& \leq c+\frac{1}{2}\left\|\partial_{t} g_{\delta}\right\|_{L^{2}(0, T ; H)}^{2}+\left(\frac{1}{2}+K\right) \int_{0}^{t} \int_{\Omega}\left|\partial_{t} u_{\delta}\right|^{2} .
\end{aligned}
$$

As a result, we obtain the following estimate

$$
\left\|\mu_{\delta}\right\|_{L^{\infty}\left(0, T ; V_{0}\right)}+\left\|u_{\delta}\right\|_{W^{1, \infty}(0, T ; H)}+\left\|\widehat{\beta^{-1}}\left(\xi_{\delta}\right)\right\|_{L^{\infty}\left(0, T ; L^{1}(\Omega)\right)}+\sqrt{\delta}\left\|u_{\delta}\right\|_{H^{1}(0, T ; V)} \leq c
$$

whence, by comparison in (2.16), the inequality

$$
\left\|\mu_{\delta}\right\|_{L^{\infty}\left(0, T ; W_{0}\right)} \leq c
$$

\subsection{Third estimate}

The purpose of this subsection is to show that if the initial data satisfies the boundedness assumption (2.39) then $u_{\delta}$ stays bounded in an interval $\left[a_{0}^{\prime}, b_{0}^{\prime}\right] \subset(a, b)$ uniformly in $\delta$, namely

$$
a_{0}^{\prime} \leq u_{\delta} \leq b_{0}^{\prime}, \quad \text { a.e. in } Q,
$$

with $a_{0}^{\prime}, b_{0}^{\prime} \in \mathbb{R}$ independent of $\delta$ and $\left[a_{0}, b_{0}\right] \subseteq\left[a_{0}^{\prime}, b_{0}^{\prime}\right] \subset(a, b)$. The idea here is to apply the maximum principle to a nonlinear elliptic system that arises from a time-discretization of (1.2), as in Section 3 .

To this end, let us note that, thanks to the estimate (5.11), the continuous embedding $W_{0} \hookrightarrow L^{\infty}(\Omega)$ and assumption (2.41), there exists a positive constant $M$, independent of $\delta$ such that

$$
\left\|\mu_{\delta}-g_{\delta}\right\|_{L^{\infty}(Q)} \leq M
$$

Now, in principle the constants $a_{0}^{\prime}$ and $b_{0}^{\prime}$ given by Theorem 2.2 may depend on $\delta$. However, going back to Section 3 , we note that the choice of the constants $\bar{a}, \bar{b}, a_{0}^{\prime}, b_{0}^{\prime}$ only depends 
on $\left\|\mu_{\delta \lambda}-g_{\delta}\right\|_{L^{\infty}(Q)}$ and the behaviour of $\psi$. Hence, the uniform estimate (5.12) implies that $a_{0}^{\prime}$ and $b_{0}^{\prime}$ can be chosen independently of the parameter $\delta$.

As a consequence, we deduce that there exist $a_{0}^{\prime}, b_{0}^{\prime} \in \mathbb{R}$, independent of $\delta$, with $\left[a_{0}, b_{0}\right] \subseteq\left[a_{0}^{\prime}, b_{0}^{\prime}\right] \subset(a, b)$, such that

$$
a_{0}^{\prime} \leq u_{\delta} \leq b_{0}^{\prime} \quad \text { a.e. in } Q, \quad \forall \delta>0 .
$$

Hence, since $\psi^{\prime} \in C^{1}\left(\left[a_{0}^{\prime}, b_{0}^{\prime}\right]\right)$, we also have

$$
\left\|\psi^{\prime}\left(u_{\delta}\right)\right\|_{L^{\infty}(Q)}+\left\|\psi^{\prime \prime}\left(u_{\delta}\right)\right\|_{L^{\infty}(Q)} \leq c .
$$

Arguing now as in Section 3 in order to prove (3.19) - 3.20), i.e. formally testing (2.17) by $-\delta^{1 / 2} \Delta \partial_{t} u_{\delta}$, we have by the Young inequality and estimate (5.14) that

$$
\begin{aligned}
\varepsilon \delta^{1 / 2} \int_{Q_{t}}\left|\nabla \partial_{t} u_{\delta}\right|^{2}+\delta^{1 / 2} \int_{Q_{t}} \nabla \xi_{\delta} \cdot \nabla \partial_{t} u_{\delta}+\frac{\delta^{3 / 2}}{2} \int_{\Omega}\left|\Delta u_{\delta}(t)\right|^{2} \\
\leq \frac{\delta^{3 / 2}}{2} \int_{\Omega}\left|\Delta u_{0 \delta}\right|^{2}-\delta^{1 / 2} \int_{0}^{t} V^{*}\left\langle\Delta \partial_{t} u_{\delta}, \mu_{\delta}-\psi^{\prime}\left(u_{\delta}\right)-g_{\delta}\right\rangle_{V} \\
=\frac{\delta^{3 / 2}}{2} \int_{\Omega}\left|\Delta u_{0 \delta}\right|^{2}+\int_{Q_{t}}\left(\nabla \mu_{\delta}-\psi^{\prime \prime}\left(u_{\delta}\right) \nabla u_{\delta}-\nabla g_{\delta}\right) \cdot\left(\delta^{1 / 2} \nabla \partial_{t} u_{\delta}\right) \\
\leq c\left(\delta^{3 / 2} \int_{\Omega}\left|\Delta u_{0 \delta}\right|^{2}+\left\|\mu_{\delta}\right\|_{L^{2}\left(0, T ; V_{0}\right)}^{2}+\delta^{1 / 2}\left\|g_{\delta}\right\|_{L^{2}(0, T ; V)}^{2}\right) \\
\quad+\frac{\varepsilon \delta^{1 / 2}}{2} \int_{Q_{t}}\left|\nabla \partial_{t} u_{\delta}\right|^{2}+c \delta^{1 / 2} \int_{Q_{t}}\left|\nabla u_{\delta}\right|^{2}
\end{aligned}
$$

Hence, rearranging the terms, using the assumptions (2.37)-(2.41), together with the monotonicity of $\beta$, we infer that

$$
\frac{\varepsilon}{2} \delta^{1 / 2} \int_{Q_{t}}\left|\nabla \partial_{t} u_{\delta}\right|^{2}+\frac{\delta^{3 / 2}}{2} \int_{\Omega}\left|\Delta u_{\delta}(t)\right|^{2} \leq c\left(1+\delta^{1 / 2} \int_{Q_{t}}\left|\nabla u_{\delta}\right|^{2}\right) .
$$

Writing $u_{\delta}=u_{0 \delta}+\int_{0}^{\cdot} \partial_{t} u_{\delta}(s) d s$ and recalling the assumption (2.40), we deduce that (updating the constant $c$ at each step)

$$
\begin{aligned}
& \frac{\varepsilon \delta^{1 / 2}}{2} \int_{Q_{t}}\left|\nabla \partial_{t} u_{\delta}\right|^{2}+\frac{\delta^{3 / 2}}{2} \int_{\Omega}\left|\Delta u_{\delta}(t)\right|^{2} \\
& \leq c\left(1+2 \delta^{1 / 2}\left\|\nabla u_{0 \delta}\right\|_{H}^{2}+2 T \delta^{1 / 2} \int_{0}^{t} \int_{Q_{s}}\left|\nabla \partial_{t} u_{\delta}\right|^{2} d s\right) \\
& \leq c\left(1+\delta^{1 / 2} \int_{0}^{t} \int_{Q_{s}}\left|\nabla \partial_{t} u_{\delta}\right|^{2} d s\right) .
\end{aligned}
$$

Hence, by the Gronwall lemma we deduce also the estimate

$$
\delta^{1 / 4}\left\|\nabla \partial_{t} u_{\delta}\right\|_{L^{2}(0, T ; H)}+\delta^{3 / 4}\left\|\Delta u_{\delta}\right\|_{L^{\infty}(0, T ; H)} \leq c,
$$

and, by comparison in (2.17),

$$
\left\|\xi_{\delta}\right\|_{L^{\infty}(0, T ; H)} \leq c .
$$




\subsection{Passage to the limit}

From the a priori estimates (5.9) $-(\underline{5.16)}$ ), using standard compactness results we have the following convergences, up to a subsequence,

$$
\begin{aligned}
u_{\delta} \stackrel{*}{\rightarrow} u & \text { in } W^{1, \infty}(0, T ; H) \cap L^{\infty}(Q), \\
\mu_{\delta} \stackrel{*}{\rightarrow} \mu & \text { in } L^{\infty}\left(0, T ; W_{0}\right) \\
\delta^{1 / 2} u_{\delta} \rightarrow 0 & \text { in } H^{1}(0, T ; V) \\
\delta u_{\delta} \rightarrow 0 & \text { in } L^{\infty}\left(0, T ; W_{\mathbf{n}}\right) \\
\xi_{\delta} \stackrel{*}{\rightarrow} \xi & \text { in } L^{\infty}(0, T ; H) .
\end{aligned}
$$

In order to pass to the limit in the equation (2.17), we need to prove now a strong convergence for the sequence $\left(u_{\delta}\right)_{\delta}$. We take the difference of (2.16)-(2.17) for two different indexes $\delta$ and $\delta^{\prime}$ : then, we test the first equation by $\mu_{\delta}-\mu_{\delta^{\prime}}$ and the second by $\partial_{t}\left(u_{\delta}-u_{\delta^{\prime}}\right)$, obtaining

$$
\begin{aligned}
& \int_{Q_{t}}\left|\nabla\left(\mu_{\delta}-\mu_{\delta^{\prime}}\right)\right|^{2}+\varepsilon \int_{Q_{t}}\left|\partial_{t}\left(u_{\delta}-u_{\delta^{\prime}}\right)\right|^{2}+\int_{Q_{t}}\left(\xi_{\delta}-\xi_{\delta^{\prime}}\right)\left(\partial_{t} u_{\delta}-\partial_{t} u_{\delta^{\prime}}\right) \\
& \leq S_{\delta, \delta^{\prime}}(t)-\int_{Q_{t}}\left(\psi^{\prime}\left(u_{\delta}\right)-\psi^{\prime}\left(u_{\delta^{\prime}}\right)\right) \partial_{t}\left(u_{\delta}-u_{\delta^{\prime}}\right)
\end{aligned}
$$

where

$$
S_{\delta, \delta^{\prime}}(t):=\int_{Q_{t}}\left(\delta \Delta u_{\delta}-\delta^{\prime} \Delta u_{\delta^{\prime}}\right) \partial_{t}\left(u_{\delta}-u_{\delta^{\prime}}\right)-\int_{Q_{t}}\left(g_{\delta}-g_{\delta^{\prime}}\right) \partial_{t}\left(u_{\delta}-u_{\delta^{\prime}}\right) .
$$

Note that by (5.15) and (2.42) we have that $\left(S_{\delta, \delta^{\prime}}\right)_{\delta, \delta^{\prime}}$ is uniformly bounded in $W^{1, \infty}(0, T)$ and converges pointwise to 0 due to the convergences above. Hence, we deduce that

$$
S_{\delta, \delta^{\prime}} \rightarrow 0 \quad \text { in } C^{0}([0, T])
$$

Moreover, since $\psi^{\prime} \in C^{1}\left(\left[a_{0}^{\prime}, b_{0}^{\prime}\right]\right)$, we have

$$
\begin{aligned}
& \int_{Q_{t}}\left|\psi^{\prime}\left(u_{\delta}\right)-\psi^{\prime}(u)\right|\left|\partial_{t}\left(u_{\delta}-u_{\delta^{\prime}}\right)\right| \\
& \leq \frac{\varepsilon}{2} \int_{Q}\left|\partial_{t}\left(u_{\delta}-u_{\delta^{\prime}}\right)\right|^{2}+\frac{1}{2 \varepsilon}\left\|\psi^{\prime \prime}\right\|_{L^{\infty}\left(a_{0}^{\prime}, b_{0}^{\prime}\right)}^{2} \int_{Q_{t}}\left|u_{\delta}-u_{\delta^{\prime}}\right|^{2},
\end{aligned}
$$

where

$$
\int_{Q_{t}}\left|u_{\delta}-u_{\delta^{\prime}}\right|^{2} \leq 2 T\left\|u_{0 \delta}-u_{0 \delta^{\prime}}\right\|_{H}^{2}+2 T \int_{0}^{t} \int_{Q_{s}}\left|\partial_{t}\left(u_{\delta}-u_{\delta^{\prime}}\right)\right|^{2} d s .
$$

Hence, rearranging the terms and using the monotonicity of $\beta$ and (2.15), we have that

$$
\begin{aligned}
& \int_{Q_{t}}\left|\nabla\left(\mu_{\delta}-\mu_{\delta^{\prime}}\right)\right|^{2}+\frac{\varepsilon}{2} \int_{Q_{t}}\left|\partial_{t}\left(u_{\delta}-u_{\delta^{\prime}}\right)\right|^{2} \\
& \leq\left\|S_{\delta, \delta^{\prime}}\right\|_{L^{\infty}(0, T)}+c\left(\left\|u_{0 \delta}-u_{0}\right\|_{H}^{2}+\int_{0}^{t} \int_{Q_{s}}\left|\partial_{t}\left(u_{\delta}-u\right)\right|^{2} d s\right)
\end{aligned}
$$


for a positive constant $c$, independent of $\delta$ and $\delta^{\prime}$. The Gronwall lemma yields then

$$
\left\|\mu_{\delta}-\mu_{\delta^{\prime}}\right\|_{L^{2}\left(0, T ; V_{0}\right)}^{2}+\left\|\partial_{t}\left(u_{\delta}-u_{\delta^{\prime}}\right)\right\|_{L^{2}(0, T ; H)}^{2} \leq c\left(\left\|S_{\delta, \delta^{\prime}}\right\|_{L^{\infty}(0, T)}+\left\|u_{0 \delta}-u_{0 \delta^{\prime}}\right\|_{H}^{2}\right)
$$

possibly updating the value of $c$. Recalling again (2.42), we deduce that

$$
u_{\delta} \rightarrow u \quad \text { in } H^{1}(0, T ; H), \quad \mu_{\delta} \rightarrow \mu \quad \text { in } L^{2}\left(0, T ; V_{0}\right) .
$$

In particular, we have the convergence

$$
\int_{Q}\left|\psi^{\prime}\left(u_{\delta}\right)-\psi^{\prime}(u)\right|^{2} \leq\left\|\psi^{\prime \prime}\right\|_{L^{\infty}\left(a_{0}^{\prime}, b_{0}^{\prime}\right)}^{2} \int_{Q}\left|u_{\delta}-u\right|^{2} \rightarrow 0 .
$$

Therefore, the strong convergence of $u_{\delta}$ and the weak convergence of $\xi_{\delta}$ to $\xi$ allow us to prove (2.28), i.e. the inclusion $\xi \in \beta\left(\partial_{t} u\right)$, by maximal monotonicity. Then, passing to the limit in (2.16) $-(2.18)$ as $\delta \searrow 0$, we can conclude.

Finally, note that letting $\delta^{\prime} \searrow 0$ in (5.17) and taking (5.15) into account, by the Young inequality we obtain

$$
\left\|\mu_{\delta}-\mu\right\|_{L^{2}\left(0, T ; V_{0}\right)}^{2}+\left\|\partial_{t}\left(u_{\delta}-u\right)\right\|_{L^{2}(0, T ; H)}^{2} \leq c\left(\left\|u_{0 \delta}-u_{0}\right\|_{H}^{2}+\left\|g_{\delta}-g\right\|_{L^{2}(0, T ; H)}^{2}+\delta^{1 / 2}\right)
$$

that is nothing but (2.50). Thus, we conclude the proof of Theorem 2.6.

\section{Acknowledgments}

EB and PC gratefully acknowledge some financial support from the GNAMPA (Gruppo Nazionale per l'Analisi Matematica, la Probabilità e le loro Applicazioni) of INdAM (Istituto Nazionale di Alta Matematica) and the IMATI - C.N.R. Pavia. Moreover, PC recognizes the contribution by the Italian Ministry of Education, University and Research (MIUR): Dipartimenti di Eccellenza Program (2018-2022) - Dept. of Mathematics "F. Casorati", University of Pavia. LS has been funded by the Vienna Science and Technology Fund (WWTF) through Project MA14-009. GT acknowledges the support of INdAM's GNFM (Gruppo Nazionale per la Fisica Matematica) and the Grant of Excellence Departments, MIUR-Italy (Art.1, commi 314-337, Legge 232/2016).

\section{References}

[1] A. Agosti, P.F. Antonietti, P. Ciarletta, M. Grasselli, and M. Verani. A Cahn-Hilliardtype equation with application to tumor growth dynamics. Math. Methods Appl. Sci. 40 (2017), 7598-7626.

[2] F. Bagagiolo and A. Visintin. Hysteresis in filtration through porous media. Z. Anal. Anwendungen 19 (2000), 977-997.

[3] V. Barbu. "Nonlinear differential equations of monotone types in Banach spaces". Springer Monographs in Mathematics. Springer, New York (2010). 
[4] E. Bonetti, P. Colli and G. Tomassetti. A non-smooth regularization of a forwardbackward parabolic equation. Math. Models Methods Appl. Sci. 27 (2017), 641-661.

[5] E. Bonetti, P. Colli, L. Scarpa and G. Tomassetti. A doubly nonlinear Cahn-Hilliard system with nonlinear viscosity. Commun. Pure Appl. Anal. 17 (2018), 1001-1022.

[6] A.L. Bertozzi, S.Esedoglu, and A. Gillette. Inpainting of binary images using the Cahn-Hilliard equation. IEEE Trans. Image Process. 16 (2007), 285-291.

[7] N.D. Botkin, M. Brokate and E. El Behi-Gornostaeva. One-phase flow in porous media with hysteresis. Physica B 486 (2016), 183-186.

[8] J.W. Cahn. On spinodal decomposition. Acta Metall. 9 (1961), 795-801.

[9] J.W. Cahn and J.E. Hilliard. Free energy of a nonuniform system. I. Interfacial free energy. J. Chem. Phys. 28 (1958), 258-267.

[10] P. Colli, G. Gilardi, P. Podio-Guidugli and J. Sprekels. Well-posedness and long-time behavior for a nonstandard viscous Cahn-Hilliard system. SIAM J. Appl. Math. 71 (2011), 1849-1870.

[11] P. Colli, G. Gilardi, P. Podio-Guidugli and J. Sprekels. Global existence and uniqueness for a singular/degenerate Cahn-Hilliard system with viscosity. J. Differential Equations 254 (2013), 4217-4244.

[12] P. Colli, G. Gilardi, E. Rocca and J. Sprekels. Vanishing viscosities and error estimate for a Cahn-Hilliard type phase field system related to tumor growth. Nonlinear Anal. Real World Appl. 26 (2015), 93-108.

[13] P. Colli, G. Gilardi, E. Rocca and J. Sprekels. Asymptotic analyses and error estimates for a Cah-Hilliard type phase field system modelling tumor growth. Discrete Contin. Dyn. Syst. Ser. S 10 (2017), 37-54.

[14] P. Colli and L. Scarpa. From the viscous Cahn-Hilliard equation to a regularized forward-backward parabolic equation. Asymptot. Anal. 99 (2016), 183-205.

[15] P.C. Fife. Models for phase separation and their mathematics. Electron. J. Differential Equations 48 (2000), 26 pp.

[16] C.G. Gal, M. Grasselli and A. Miranville. Cahn-Hilliard-Navier-Stokes systems with moving contact lines. Calc. Var. Partial Differential Equations 55 (2016), Art. 50, $47 \mathrm{pp}$.

[17] M.E. Gurtin. Generalized Ginzburg-Landau and Cahn-Hilliard equations based on a microforce balance. Phys. D 92 (1996), 178-192.

[18] M. Latroche. Structural and thermodynamic properties of metallic hydrides used for energy storage. J. Phys. Chem. Solids 65 (2004) 517-522.

[19] J. L. Lions. "Quelques méthodes de résolution des problèmes aux limites non linéaires". Dunod; Gauthier-Villars, Paris (1969). 
[20] Q.X. Liu, M. Rietkerk, P.M.J. Herman, T. Piersma, J.M. Fryxell, and J. van de Koppel. Phase separation driven by density-dependent movement: A novel mechanism for ecological patterns. Phys. Life Rev., 19 (2016) 107-121.

[21] A. Miranville. Some generalizations of the Cahn-Hilliard equation. Asymptot. Anal. 22 (2000) 235-259.

[22] A. Miranville and G. Schimperna. On a doubly nonlinear Cahn-Hilliard-Gurtin system. Discrete Contin. Dyn. Syst. Ser. B 14 (2010), 675-697.

[23] A. Miranville and S. Zelik. Doubly nonlinear Cahn-Hilliard-Gurtin equations. Hokkaido Math. J. 38 (2009), 315-360.

[24] A. Miranville. The Cahn-Hilliard equation and some of its variants. AIMS Math. 2 (2017), 479-544.

[25] A. Novick-Cohen. On the viscous Cahn-Hilliard equation, in "Material instabilities in continuum mechanics (Edinburgh, 1985-1986)". Oxford Sci. Publ., Oxford Univ. Press, New York, (1988), 329-342.

[26] A. Novick-Cohen and R. L. Pego. Stable patterns in a viscous diffusion equation. Trans. Amer. Math. Soc. 324 (1991), 331-351.

[27] P. Podio-Guidugli. Models of phase segregation and diffusion of atomic species on a lattice. Ric. Mat. 55 (2006), 105-118.

[28] T. Roubíček. "Nonlinear partial differential equations with applications". Birkhäuser Verlag, Basel (2005).

[29] L. Scarpa. Existence and uniqueness of solutions to singular Cahn-Hilliard equations with nonlinear viscosity terms and dynamic boundary conditions. J. Math. Anal. Appl. 469 (2019), 730-764.

[30] B. Schweizer. Hysteresis in porous media: modelling and analysis. Interfaces Free Bound. 19 (2017), 417-447.

[31] J. Simon. Compact sets in the space $L^{p}(0, T ; B)$. Ann. Mat. Pura Appl. (4) 146 (1987), 65-96.

[32] G. Tomassetti. Smooth and non-smooth regularizations of the nonlinear diffusion equation. Discrete Contin. Dyn. Syst. Ser. S 10 (2017) 1519-1537. 